\title{
A global checklist of the 932 fruit fly species in the tribe Dacini (Diptera, Tephritidae)
}

\author{
Camiel Doorenweerd', Luc Leblanc², Allen L. Norrbom³ \\ Michael San Jose', Daniel Rubinoff'
}

I University of Hawaii, Department of Plant and Environmental Protection Services, 3050 Maile Way, Honolulu, Hawaii, 96822-2231, USA 2 University of Idaho, Department of Entomology, Plant Pathology and Nematology, 875 Perimeter Drive, MS2329, Moscow, Idaho, 83844-2329, USA 3 Systematic Entomology Laboratory, ARS, USDA, clo Smithsonian Institution, P.O. Box 37012, MRC 168, Washington, DC 20013-7012, USA

Corresponding author: Camiel Doorenweerd (camiel.doorenweerd@hawaii.edu)

Academic editor: M. De Meyer | Received 20 October 2017 | Accepted 14 December 2017 | Published 17 January 2018

http://zoobank.org/F1BF770B-254D-4F8F-976B-A2F53A7E4A60

Citation: Doorenweerd C, Leblanc L, Norrbom AL, Jose MS, Rubinoff D (2018) A global checklist of the 932 fruit fly species in the tribe Dacini (Diptera, Tephritidae). ZooKeys 730: 19-56. https://doi.org/10.3897/zookeys.730.21786

\begin{abstract}
The correct application of the scientific names of species is neither easy nor trivial. Mistakes can lead to the wrong interpretation of research results or, when pest species are involved, inappropriate regulations and limits on trade, and possibly quarantine failures that permit the invasion of new pest species. Names are particularly challenging to manage when groups of organisms encompass a large number of species, when different workers employ different philosophical views, or when species are in a state of taxonomic flux. The fruit fly tribe Dacini is a species-rich taxon within Tephritidae and contains around a fifth of all known species in the family. About $10 \%$ of the 932 currently recognized species are pests of commercial fruits and vegetables, precipitating quarantines and trade embargos. Authoritative species lists consist largely of scattered regional treatments and outdated online resources. The checklist presented here is the first global overview of valid species names for the Dacini in almost two decades, and includes new lure records. By publishing this list both in paper and digitally, we aim to provide a resource for those studying fruit flies as well as researchers studying components of their impact on agriculture. The list is largely a consolidation of previous works, but following the results from recent phylogenetic work, we transfer one subgenus and eight species to different genera: members of the Bactrocera subgenus Javadacus Hardy, considered to belong to the Zeugodacus group of subgenera, are transferred to genus Zeugodacus; Bactrocera pseudocucurbitae White, 1999, stat. rev., is transferred back to Bactrocera from Zengodacus; Zeugodacus arisanicus Shiraki, 1933, stat. rev., is transferred back to Zeugodacus from Bactrocera; and $Z$.
\end{abstract}

Copyright Camiel Doorenweerd et al. This is an open access article distributed under the terms of the Creative Commons Attribution License (CC BY 4.0), which permits unrestricted use, distribution, and reproduction in any medium, provided the original author and source are credited. 
brevipunctatus (David \& Hancock, 2017), comb. n.; Z. javanensis (Perkins, 1938), comb. n.; Z. montanus (Hardy, 1983), comb. n.; Z. papuaensis (Malloch, 1939), comb. n.; Z. scutellarius (Bezzi, 1916), comb. n.; Z. semisurstyli (Drew \& Romig, 2013), comb. n.; and Z. trilineatus (Hardy, 1955), comb. n. are transferred from Bactrocera to Zeugodacus.

\section{Keywords}

global, pest, cryptic, Bactrocera, Zeugodacus, Dacus

\section{Introduction}

Despite the current 'phylogenomic' age and the generation of large amounts of data on relatively few, selected, organisms, discovering and classifying new species is an ongoing endeavor of basic science that is far from complete (Zhang 2011). Major challenges to advance taxonomic work lie, among others, in the correct application of scientific species names, which in turn depends on the availability of accurate reference databases. Global initiatives to provide reference lists of species names (e.g., Roskov et al. 2017) all include major gaps that can only be filled by taxonomic specialists. Some groups of organisms are particularly challenging to manage because of the number of species they encompass, conceptual differences between workers, or the existence of unresolved problems with species identities or concepts themselves. Simultaneously, those same groups will likely benefit the most from an authoritative overview.

The fruit fly tribe Dacini is a species-rich radiation within Tephritidae and contains around a fifth of all known species in the family (Norrbom et al. 1999, Pape et al. 2011, Schutze et al. 2017). All Dacini members are frugivorous or florivorous and about $10 \%$ of the 932 currently recognized species are pests of commercial fruits and vegetables (Fletcher 1987, White and Elson-Harris 1992, Vargas et al. 2015, Freidberg et al. 2017). Among these are some of the world's economically most important pests, such as the widely introduced oriental fruit fly, Bactrocera dorsalis (Hendel, 1912), carambola fruit fly Bactrocera carambolae Drew \& Hancock, 1994, and the melon fly, Zengodacus cucurbitae (Coquillett, 1899) (De Meyer et al. 2015, Ekesi et al. 2016). The tribe as a whole has received considerable taxonomic attention and new species are continuously being discovered (Fig. 1; Leblanc et al. 2015a, David et al. 2016, 2017). Dacini flies are phenotypically very similar and therefore also one of the most difficult groups of Tephritidae to identify to species-level. Whereas many Tephritidae can be identified from their intricate wing patterns, which are commonly thought to have evolved to deter predators (such as Salticidae jumping spiders [Whitman et al. 1988]), for mating rituals, or territorial behavior, the wings of most Dacini are clear with only a costal band and, usually, an anal streak. The adult chaetotaxy is a set of characters that is usually of value in dipteran species identification, but in Dacini the number of setae is reduced and similar configurations may often be homoplaseous (Hardy 1955, Hancock and Drew 2015). Their body colors, various combinations of black and yellow to red, are commonly thought to have resulted from wasp mimicry and may be under se- 


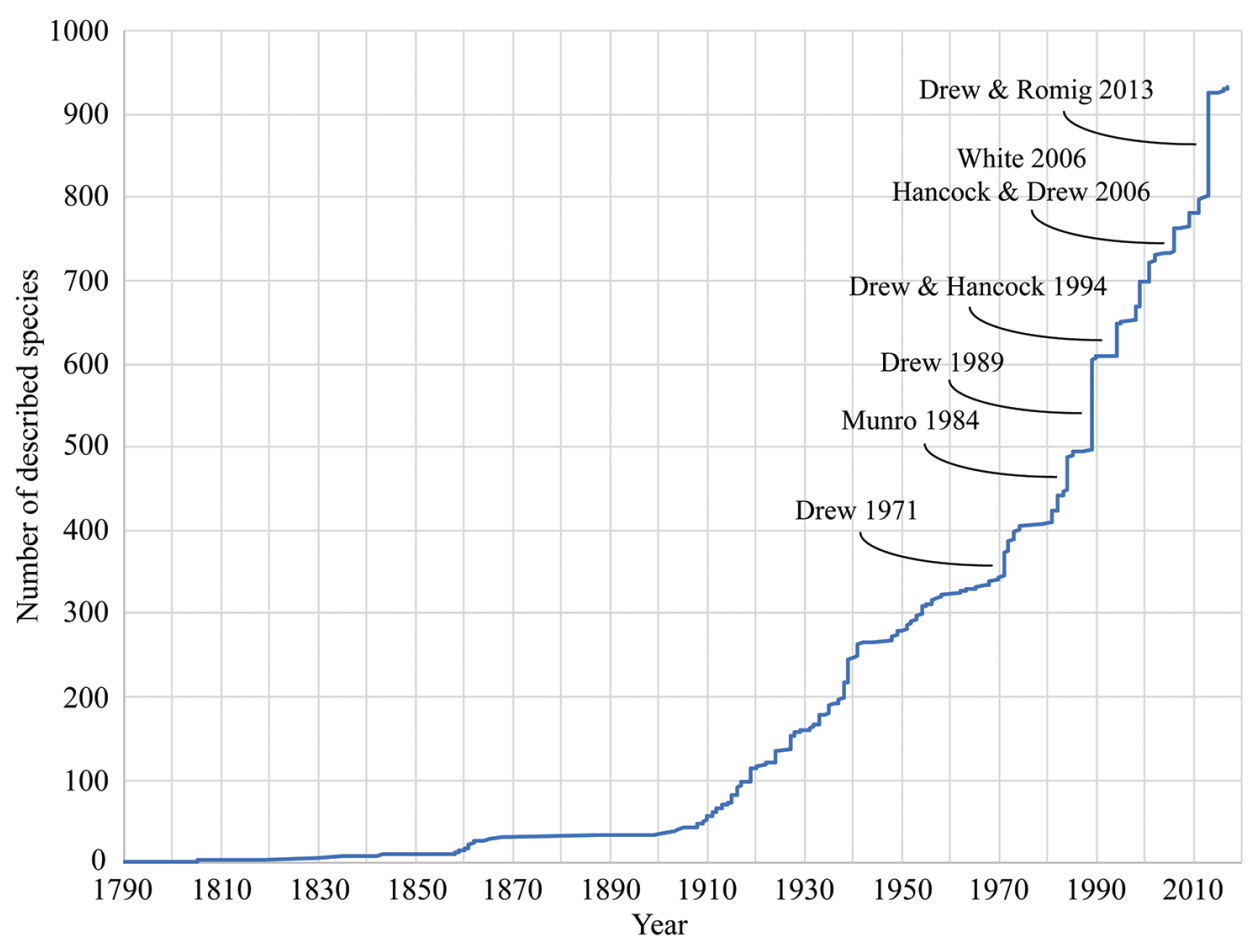

Figure I. Accumulation of described species in Dacini through time, with publications that featured large numbers of newly described species indicated at their respective moment. The first species was described in 1790, but during the past fifty years the number of recognized [or known] species has more than doubled to reach the current 932 .

lective pressure (White 2006). Diagnostic body color patterns used to separate species are further confounded by considerable intraspecific variation (Leblanc et al. 2015b). The combination of these factors has resulted in a long history of unstable classification and even though molecular phylogenetic studies are now reaching a general consensus, this has not fully trickled down to the nomenclatural level.

Dacini is a tropical and subtropical evolutionary radiation of flies with centers of diversity in Southeast Asia and Sub-Saharan Africa. Early molecular phylogenetic studies focused on pest species, often of a particular region, leading to biased results on the relationships between species that may not accurately reflect monophyletic origins or sister-group assignments (Smith et al. 2003, Nakahara and Muraji 2008, Virgilio et al. 2015). With phylogenetic studies expanding their scope beyond the pest species and increased use of molecular data, it became clear that the initial morphology-based classifications had to be revised and, in particular, the large genus Bactrocera had to be split into Bactrocera and Zeugodacus because the latter is more closely related to Dacus (Krosch et al. 2012, Virgilio et al. 2015, San Jose et al. 2018 in press, Dupuis et al. 2017). Following the most recent results, there are currently four genera in Dacini: Monacrostichus Bezzi, Dacus Fabricius, Bactrocera Macquart and Zeugodacus Hendel 
(De Meyer et al. 2015, Virgilio et al. 2015, Freidberg et al. 2017), although some authors (e.g., Drew and Romig 2013, 2016, Hancock and Drew 2016) continue to include Zeugodacus within Bactrocera. Ichneumonopsis Hardy is now placed in Gastrozonini (Norrbom et al. 1999, Freidberg et al. 2017). Aside from shifts in generic assignments, taxa have been variably assigned to species complexes, species groups, subgenera and species-complex groups to provide some additional systematic structure, primarily for the purpose of identification keys (Clarke et al. 2005, White 2006, Drew and Romig 2013). These intermediate taxonomic ranks are mostly groups of convenience defined by unique combinations of characters rather than by synapomorphic characters. The largest and most intesively studied is the Bactrocera dorsalis complex with 88 species; the group that, incidentally, also holds the largest number of pest species. This complex, like most others, is not monophyletic (Leblanc et al. 2015b, Virgilio et al. 2015, San Jose et al. 2018 in press) and there has been synonymy of several significant pest species, such as B. papayae Drew \& Hancock, 1994, and B. invadens Drew, Tsuruta \& White, 2005 with B. dorsalis (San Jose et al. 2013, Schutze et al. 2015a, 2015b). To facilitate communication and progress of our understanding of the group, a reliable taxonomic starting point is badly needed and will enable further studies into the taxonomy and systematics of the tribe.

The most recently published catalogs that covered Dacini globally are now almost two decades old (Norrbom et al. 1999, Norrbom 2004) and scattered regional treatments and keys currently comprise the largest body of references for Dacini. For Southeast Asia, there is a relatively recent two-part work including a revision (Drew and Romig 2013) and the accompanying keys that followed (Drew and Romig 2016). These books have incorporated the previous keys for the $B$. dorsalis complex of Drew and Hancock (1994), but they did not adopt the latest results from a series of molecular phylogenetic works, including the split of Bactrocera into Bactrocera and Zeugodacus. For other regions, all treatments are older with increased confusion due to differing morphological terminology, species designations, and assignments. For Africa, the most recent works are two treatments from 2006 (Hancock and Drew 2006, White 2006), and for Australasia there is a treatment from 1989 (Drew 1989), including keys, a proposed subgeneric classification, and revisions for the species in the region. As of 2017, the Drew and Romig books on the Asian fauna are in print and available for sale, and the other works are available digitally online and provide important catalogue references. Online resources that aim to provide up-todate species checklists such as Systema Dipterorum (Pape and Thompson 2013), the Catalogue of Life (Roskov et al. 2017), or the pest-oriented database of the Centre for Agriculture and Biosciences (CABI 2017) are outdated and have not been able to combine the regional treatments appropriately. Valid and invalid names can be verified using the Tephritidae Databases on the COFFHI website (https://coffhi. cphst.org/), but it was primarily designed for host plant information and the tephritid name searches are currently undergoing revision. Other websites, such as the "True Fruit Flies of the Afrotropical Region" (De Meyer and White 2016) or the "PACIFLY" website, covering the Pacific region (Pest Management in the Pacific Project 2003), 
contain valuable information, but are limited in scope and are irregularly maintained due to sporadic funding. The checklist presented here is a global overview of valid species names of Dacini. By publishing this list in paper and digital format we hope to provide a resource for those studying fruit fly taxonomy as well as researchers concerned with their impacts on agriculture. The list is largely a consolidation of previous works, but following the results from recent phylogenetic work (Virgilio et al. 2015, San Jose et al. 2018 in press), we transfer one subgenus and eight species to different genera: Bactrocera subgenus Javadacus Hardy, considered to belong to the Zeugodacus group of subgenera by Hancock and Drew (2017), is transferred to genus Zeugodacus; Bactrocera pseudocucurbitae White, 1999, stat. rev., is transferred back to Bactrocera from Zeugodacus; Zeugodacus arisanicus Shiraki, 1933, stat. rev., is transferred back to Zeugodacus from Bactrocera; and Z. brevipunctatus (David \& Hancock, 2017a), comb. n., Z. javanensis (Perkins, 1938), comb. n., Z. montanus (Hardy, 1983), comb. n., Z. papuaensis (Malloch, 1939), comb. n., Z. scutellarius (Bezzi, 1916), comb. n., Z. semisurstyli (Drew \& Romig, 2013), comb. n., and Z. trilineatus (Hardy, 1955), comb. n. are transferred from Bactrocera to Zeugodacus.

\section{Methods}

\section{Checklist}

The source data is, for a large part, comprised of regional treatments (Drew 1989, Hancock and Drew 2006, White 2006, Drew and Romig 2013), with additions and revisions from more recent studies (Drew et al. 2011, Yu et al. 2012, Hancock 2015, Hancock and Drew 2015, Hendrichs et al. 2015, Schutze et al. 2015b, David et al. 2016, 2017, Drew and Hancock 2016, Freidberg et al. 2017, Han et al. 2017). Species included in the list are ordered alphabetically by genus. We do not indicate subgeneric or species complex ranks because their biological significance is, at present, unclear (Leblanc et al. 2015b). We do, however, provide the checklist also in spreadsheet form in supplementary material (S1) where these ranks are included and users can sort the species to their preference. For distribution, we use a coarse geographical indication: African or Asia-Pacific. The native region is indicated in the line with the species name, invasive regions are mentioned in the notes. We also include known male lure records for each species: cue-lure, methyl eugenol, isoeugenol and zingerone. Zingerone, first reported as a male lure by Tan and Nishida (2000), has received increased attention in recent years, with new lure records, including species not attracted to the two other lures, for a number of species in Australia and Papua New Guinea (Fay 2012, Royer et al. 2015, 2017). These records are included in the list, along with previously unpublished new records, indicated as such, from our team's recent surveys carried out in Taiwan, Vietnam, Sri Lanka, Bangladesh and Nepal. For morphological terminology we follow White et al. (2000), which follows that in standard usage for other Diptera and differs somewhat from the older treatments. 


\section{Conflicting views}

For some species that have recently been synonymized or where there are conflicting views by different authors, we have indicated this under the 'notes' for the respective species, so that this may help users to place different views in perspective. It should also be noted that some authors do not follow the elevation of Zeugodacus to genuslevel, because this is currently only supported by molecular data and morphological studies are inconclusive (David et al. 2017, Virgilio et al. 2015, San Jose et al. 2018). This affects the placement of nearly 200 species and although we agree that the reassignment of species may have initially been premature, recent studies corroborate the need to recognize Zeugodacus as a genus to maintain Bactrocera as monophyletic. A 168-species seven-gene phylogeny, including multiple Bactrocera subgenera, shows that Zeugodacus, Bactrocera and Dacus each are monophyletic, and provides moderate statistical support for a sister relationship between Zeugodacus and Dacus (San Jose et al. 2018). A phylogeny with less representatives from Dacini, but 878 molecular loci, provides full statistical support for the sister relationship of Zeugodacus with Dacus, and Bactrocera as sister to both (Dupuis et al. 2017). As such, Bactrocera in the old sense is paraphyletic.

\section{Gender agreement}

Because Dacini includes both masculine and feminine genera and because species have been moved between different genera over time, there is some confusion in the literature regarding the correct application of gender agreement. We have paid particular attention to this in the checklist. Most notably, several species names ending in -fer have originally been described without the author indicating if the name should be regarded as a noun or as an adjective. Following section 31.2.2 of the Zoological Code of Zoological Nomenclature (ICZN 1999), such names should be treated as a noun in apposition and the ending should not change when the species is moved to a different genus. This applies to Bactrocera terminifer (Walker, 1860), B. speculifer (Walker, 1865) and B. curvifer (Walker, 1860).

\section{Results}

An overview of the current numbers of species split per genus, worldwide and according to the region where they are native, is shown in Table 1. Five species are shared between Africa and the Asia-Pacific regions: Dacus ciliatus, Bactrocera oleae, B. dorsalis, $B$. latifrons and Zeugodacus cucurbitae. There are no Dacini native to other regions, however, some species have become invasive in various countries outside their native distribution, such as $B$. dorsalis, B. latifrons and $Z$. cucurbitae from Asia introduced to Africa and various Pacific islands, B. zonata introduced from Asia to the Indian Ocean islands and the Middle East, including Egypt, and Asian B. carambolae, that invaded 
Table I. Number of described species per region.

\begin{tabular}{l|c|c|c}
\hline & Worldwide & Africa & Asia-Pacific \\
\hline Dacini & 932 & 207 & 730 \\
\hline Bactrocera & 461 & 13 & 451 \\
\hline Zeugodacus & 196 & 1 & 195 \\
\hline Dacus & 273 & 193 & 81 \\
\hline Monacrostichus & 2 & 0 & 2 \\
\hline
\end{tabular}

the Guianas and northern Brazil in South America. Two invasive species are native to Africa; $B$. oleae was introduced to the Mediterranean area, western Asia and California and northwestern Mexico in North America, and D. ciliatus has invaded the Middle East and the Indian subcontinent (Vargas et al. 2015).

\section{Checklist}

\section{Genus Bactrocera Macquart}

Bactrocera abbreviata (Hardy, 1974). Asia-Pacific. Non-pest. Zingerone.

Notes: Zingerone is a new lure record. Bactrocera abbreviata may be a junior synonym of B. bipistulata. There are morphological intermediates from Sri Lanka in the UHIM collection with a dark facial band and darker femora.

Bactrocera abdofuscata (Drew, 1971). Asia-Pacific. Non-pest.

Bactrocera abdolonginqua (Drew, 1971). Asia-Pacific. Non-pest. Methyl eugenol.

Bactrocera abdomininigra Drew, 1989. Asia-Pacific. Non-pest.

Bactrocera abdonigella (Drew, 1971). Asia-Pacific. Non-pest. Cue-lure.

Bactrocera aberrans (Hardy, 1951). Asia-Pacific. Non-pest. Isoeugenol.

Notes: Weakly attracted to isoeugenol (Royer 2015)

Bactrocera abscondita (Drew \& Hancock, 1981). Asia-Pacific. Non-pest. Cue-lure.

Bactrocera absidata Drew, 1989. Asia-Pacific. Non-pest.

Bactrocera abundans Drew, 1989. Asia-Pacific. Non-pest. Cue-lure.

Bactrocera aceraglans White \& Evenhuis, 1999. Asia-Pacific. Non-pest.

Bactrocera aceromata White \& Evenhuis, 1999. Asia-Pacific. Non-pest.

Bactrocera aemula Drew, 1989 Asia-Pacific. Non-pest. Cue-lure.

Bactrocera aenigmatica (Malloch, 1931). Asia-Pacific. Non-pest.

Bactrocera aeroginosa (Drew \& Hancock, 1981). Asia-Pacific. Non-pest. Cue-lure, zingerone.

Bactrocera aethriobasis (Hardy, 1973). Asia-Pacific. Non-pest. Methyl eugenol.

Bactrocera affinibancroftii Drew \& Romig, 2013. Asia-Pacific. Non-pest. Methyl eugenol. Bactrocera affinidorsalis (Hardy, 1982). Asia-Pacific. Non-pest. Cue-lure.

Bactrocera affinis (Hardy, 1954). Asia-Pacific. Non-pest. Methyl eugenol.

Bactrocera aglaiae (Hardy, 1951). Asia-Pacific. Non-pest. Methyl eugenol, zingerone.

Bactrocera aithogaster Drew, 1989. Asia-Pacific. Non-pest. 
Bactrocera albistrigata de Meijere, 1911. Asia-Pacific. Fruit pest (polyphagous). Cue-lure. Notes: B. albistrigata is very similar in appearance to B. frauenfeldi. Based on UHIM collection material, the morphological variation of both species is larger than Drew and Romig (2013) suggest. Hardy (1954) considered them synonyms, but they are treated as different species in Drew and Romig (2013). Their populations are likely allopatric, but there appears to be some morphological overlap.

Bactrocera allwoodi (Drew, 1979). Asia-Pacific. Non-pest. Cue-lure.

Bactrocera alyxiae (May, 1953). Asia-Pacific. Non-pest. Cue-lure, zingerone.

Bactrocera amarambalensis Drew, 2002. Asia-Pacific. Non-pest. Methyl eugenol.

Bactrocera ampla (Drew, 1971). Asia-Pacific. Non-pest. Cue-lure.

Bactrocera amplexa (Munro, 1984). Africa. Non-pest.

Bactrocera amplexiseta (May, 1962). Asia-Pacific. Non-pest. Methyl eugenol.

Bactrocera andamanensis (Kapoor, 1971). Asia-Pacific. Non-pest. Cue-lure.

Bactrocera anfracta Drew, 1989. Asia-Pacific. Non-pest. Cue-lure.

Bactrocera angustifasciata Drew, 1989. Asia-Pacific. Non-pest.

Bactrocera anomala (Drew, 1971). Asia-Pacific. Non-pest. Cue-lure.

Bactrocera anthracina (Drew, 1971). Asia-Pacific. Non-pest. Cue-lure.

Bactrocera antigone (Drew \& Hancock, 1981). Asia-Pacific. Non-pest. Cue-lure.

Bactrocera apicofuscans White \& Tsuruta, 2001. Asia-Pacific. Non-pest. Methyl eugenol.

Bactrocera apiconigroscutella Drew, 2002. Asia-Pacific. Non-pest. Cue-lure.

Bactrocera apicopicta Drew \& Romig, 2013. Asia-Pacific. Non-pest. Cue-lure.

Bactrocera aquila (Drew, 1989). Asia-Pacific. Non-pest. Cue-lure.

Bactrocera aquilonis (May, 1965). Asia-Pacific. Fruit pest. Cue-lure.

Notes: May be a junior synonym of $B$. tryoni. The latter is supposedly restricted to the eastern side of Australia, B. aquilonis to the west. Even though these populations may now be largely allopatric, separated by the arid regions along the border between the Northern Territories and Queensland, they cannot be separated reliably based on morphology or using a population genetic approach with microsatellite data (Gilchrist et al. 2003, Cameron et al. 2010).

Bactrocera arecae (Hardy \& Adachi, 1954). Asia-Pacific. Fruit pest (monophagous).

Bactrocera assita Drew, 1989 Asia-Pacific. Non-pest. Cue-lure.

Bactrocera aterrima (Drew, 1972). Asia-Pacific. Non-pest. Cue-lure.

Bactrocera atra (Malloch, 1938). Asia-Pacific. Non-pest. Cue-lure.

Notes: Cue-lure is a new lure record from surveys in French Polynesia in 1998.

Bactrocera atrabifasciata Drew \& Romig, 2001. Asia-Pacific. Non-pest. Cue-lure.

Bactrocera atramentata (Hering, 1941). Asia-Pacific. Fruit pest (monophagous). Cuelure, zingerone.

Bactrocera atrifemur Drew \& Hancock, 1994. Asia-Pacific. Non-pest. Methyl eugenol. Bactrocera atriliniellata Drew, 1989. Asia-Pacific. Non-pest. Cue-lure.

Bactrocera aurantiaca (Drew \& Hancock, 1981). Asia-Pacific. Non-pest. Cue-lure.

Bactrocera aurea (May, 1952). Asia-Pacific. Non-pest. Zingerone.

Bactrocera avittata Drew \& Romig, 2013. Asia-Pacific. Non-pest. Cue-lure.

Bactrocera balagawii Drew, 2011. Asia-Pacific. Non-pest. Methyl eugenol. 
Bactrocera bancroftii (Tryon, 1927). Asia-Pacific. Fruit pest (oligophagous). Methyl eugenol. Bactrocera banneri White, 1999. Asia-Pacific. Non-pest.

Notes: B. banneri and B. coracina are the two members of the subgenus Perkinsidacus in the most recent treatment of these species (Hancock and Drew 2017b), and both may belong in the genus Zeugodacus. They have the shallow emargination of sternite $\mathrm{V}$ and the long surstylus lobes of the male genitalia that fit with Zeugodacus, but lack a medial vitta on the scutum and the lateral vittae do not extend anteriorly beyond the transverse suture. Because there is, at present, no molecular data to support either placement and because it is unclear which, if any, of these morphological characters are apomorphic we tentatively leave both species in Bactrocera.

Bactrocera barringtoniae (Tryon, 1927). Asia-Pacific. Non-pest.

Bactrocera batemani Drew, 1989. Asia-Pacific. Non-pest. Methyl eugenol.

Bactrocera beckerae (Hardy, 1982). Asia-Pacific. Non-pest. Cue-lure.

Bactrocera bellisi Drew \& Romig, 2013. Asia-Pacific. Non-pest. Cue-lure.

Bactrocera bhutaniae Drew \& Romig, 2013. Asia-Pacific. Non-pest. Cue-lure.

Bactrocera biarcuata (Walker, 1865). Asia-Pacific. Non-pest. Methyl eugenol.

Bactrocera bidentata (May, 1963). Asia-Pacific. Non-pest.

Bactrocera bifasciata (Hardy, 1982). Asia-Pacific. Non-pest. Cue-lure.

Bactrocera biguttula (Bezzi, 1922). Africa. Non-pest.

Bactrocera bimaculata Drew \& Hancock, 1994. Asia-Pacific. Non-pest. Cue-lure.

Bactrocera binhduongiae Drew \& Romig, 2013. Asia-Pacific. Non-pest. Methyl eugenol. Bactrocera bipustulata (Bezzi, 1914). Asia-Pacific. Non-pest. Cue-lure, zingerone.

Notes: Zingerone is a new lure record. See further comments under B. abbreviata. Bactrocera bitungiae Drew \& Romig, 2013. Asia-Pacific. Non-pest. Cue-lure.

Bactrocera bivittata Lin \& Wang, 2005. Asia-Pacific. Non-pest. Methyl eugenol. Bactrocera blairiae Drew \& Romig, 2013. Asia-Pacific. Non-pest. Methyl eugenol. Bactrocera brachycera (Bezzi, 1916). Asia-Pacific. Non-pest.

Bactrocera breviaculeus (Hardy, 1951). Asia-Pacific. Non-pest. Cue-lure, zingerone.

Bactrocera brevistriata (Drew, 1968). Asia-Pacific. Non-pest. Cue-lure.

Bactrocera bruneiae Drew \& Romig, 2013. Asia-Pacific. Non-pest. Methyl eugenol.

Bactrocera brunnea (Perkins \& May, 1949). Asia-Pacific. Non-pest.

Bactrocera brunneola White \& Tsuruta, 2001. Asia-Pacific. Non-pest. Cue-lure.

Bactrocera bryoniae (Tryon, 1927). Asia-Pacific. Fruit pest (oligophagous). Cue-lure, zingerone.

Bactrocera buinensis Drew, 1989. Asia-Pacific. Non-pest. Cue-lure.

Bactrocera bullata Drew, 1989. Asia-Pacific. Non-pest.

Bactrocera bullifera (Hardy, 1973). Asia-Pacific. Non-pest.

Bactrocera buloloensis Drew, 1989. Asia-Pacific. Non-pest.

Bactrocera cacuminata (Hering, 1941). Asia-Pacific. Non-pest. Methyl eugenol.

Bactrocera caledoniensis Drew, 1989. Asia-Pacific. Non-pest. Cue-lure.

Bactrocera caliginosa (Hardy, 1970). Asia-Pacific. Non-pest.

Bactrocera calophylli (Perkins \& May, 1949). Asia-Pacific. Non-pest.

Bactrocera captiva Drew \& Romig, 2013. Asia-Pacific. Non-pest. 
Bactrocera carambolae Drew \& Hancock, 1994. Asia-Pacific. Fruit pest (polyphagous). Methyl eugenol, zingerone.

Notes: Under laboratory conditions, B. carambolae and B. dorsalis can produce fertile F1 hybrids, though with reduced survivability, and there is evidence for hybridization in the wild. Nonetheless, based on a combination of genetic and morphological evidence, they are considered to be two separate species (Ebina and Ohto 2006, Schutze et al. 2015a). The native distribution of B. carambolae is in Southeast Asia, but it is invasive in South America (Guianas and northern Brazil). Bactrocera carbonaria (Hendel, 1927). Asia-Pacific. Non-pest. Cue-lure.

Bactrocera careofascia Drew \& Romig, 2013. Asia-Pacific. Non-pest. Cue-lure.

Bactrocera caryeae (Kapoor, 1971). Asia-Pacific. Fruit pest (polyphagous). Methyl eugenol. Bactrocera ceylanica Tsuruta \& White, 2001. Asia-Pacific. Non-pest. Cue-lure. Bactrocera cheesmanae (Perkins, 1939). Asia-Pacific. Non-pest. Methyl eugenol. Bactrocera chettalli David \& Ranganath, 2016. Asia-Pacific. Non-pest. Bactrocera cibodasae Drew \& Hancock, 1994. Asia-Pacific. Non-pest. Cue-lure. Bactrocera cinnabaria Drew \& Romig, 2013. Asia-Pacific. Non-pest. Bactrocera cinnamea Drew, 1989. Asia-Pacific. Non-pest. Cue-lure. Bactrocera circamusae Drew, 1989. Asia-Pacific. Non-pest. Cue-lure. Bactrocera citima (Hardy, 1973). Asia-Pacific. Non-pest. Cue-lure. Bactrocera cogani White, 2006. Africa. Non-pest.

Bactrocera cognata (Hardy \& Adachi, 1954). Asia-Pacific. Non-pest. Bactrocera collita Drew \& Hancock, 1994. Asia-Pacific. Non-pest. Methyl eugenol. Bactrocera commensurata Drew \& Romig, 2013. Asia-Pacific. Non-pest. Methyl eugenol. Bactrocera commina Drew, 1989. Asia-Pacific. Non-pest.

Bactrocera confluens (Drew, 1971). Asia-Pacific. Non-pest. Methyl eugenol. Bactrocera congener Drew, 1989. Asia-Pacific. Non-pest. Cue-lure.

Bactrocera consectorata Drew, 1989. Asia-Pacific. Non-pest. Cue-lure. Bactrocera contermina Drew, 1989. Asia-Pacific. Non-pest. Methyl eugenol. Bactrocera contigua Drew, 1989. Asia-Pacific. Non-pest. Methyl eugenol. Bactrocera continua (Bezzi, 1919). Asia-Pacific. Non-pest.

Bactrocera coracina (Drew, 1971). Asia-Pacific. Non-pest.

Notes: Maybe should be moved to Zeugodacus, see comments under B. banneri. Bactrocera correcta (Bezzi, 1916). Asia-Pacific. Fruit pest. (polyphagous). Methyl eugenol. Bactrocera costalis (Shiraki, 1933). Asia-Pacific. Non-pest. Cue-lure.

Bactrocera curreyi Drew, 1989. Asia-Pacific. Non-pest. Cue-lure.

Bactrocera curtivitta Drew \& Romig, 2013. Asia-Pacific. Non-pest. Bactrocera curvifer (Walker, 1864). Asia-Pacific. Non-pest. Methyl eugenol. Bactrocera curvipennis (Froggatt, 1909). Asia-Pacific. Fruit pest. Cue-lure. Bactrocera curvosterna Drew \& Romig, 2013. Asia-Pacific. Non-pest. Cue-lure. Bactrocera dapsiles Drew, 1989. Asia-Pacific. Non-pest. Methyl eugenol. Bactrocera daruensis Drew, 1989. Asia-Pacific. Non-pest. Bactrocera decumana (Drew, 1972). Asia-Pacific. Non-pest. Cue-lure. Bactrocera decurtans (May, 1965). Asia-Pacific. Non-pest. Methyl eugenol. 
Bactrocera diallagma Drew, 1989. Asia-Pacific. Non-pest. Methyl eugenol.

Bactrocera diaphana (Hering, 1953). Asia-Pacific. Non-pest.

Bactrocera digressa Radhakrishnan, 1999. Asia-Pacific. Non-pest. Cue-lure, zingerone.

Notes: Zingerone is a new lure record.

Bactrocera diospyri Drew, 1989. Asia-Pacific. Non-pest.

Bactrocera dispar (Hardy, 1982). Asia-Pacific. Non-pest.

Bactrocera distincta (Malloch, 1931). Asia-Pacific. Fruit pest. Cue-lure.

Bactrocera dongnaiae Drew \& Romig, 2013. Asia-Pacific. Non-pest. Cue-lure.

Bactrocera dorsalis (Hendel, 1912). Asia-Pacific. Fruit pest (polyphagous). Methyl eugenol, zingerone.

Notes: $B$. dorsalis, the Oriental fruit fly, is one of the most significant pest species within the Tephritidae, and it is invasive in many areas of Asia, Africa and the Pacific islands (Vargas et al. 2015). Based on a total-evidence approach, $B$. papayae, $B$. invadens and $B$. philippinensis are now considered synonyms of $B$. dorsalis, but these names can still be found in numerous papers and internet website resources. Bactrocera dorsalis is known to hybridize with $B$. carambolae and genetic evidence suggests that there is historic hybridization with $B$. kandiensis (Schutze et al. 2015b); see notes under those respective species for further details.

Bactrocera dorsaloides (Hardy \& Adachi, 1954). Asia-Pacific. Non-pest.

Bactrocera dyscrita (Drew, 1971). Asia-Pacific. Non-pest. Cue-lure.

Bactrocera ebenea (Drew, 1971). Asia-Pacific. Non-pest. Methyl eugenol.

Bactrocera ektoalangiae Drew \& Hancock, 1999. Asia-Pacific. Non-pest.

Bactrocera elongata Drew \& Romig, 2013. Asia-Pacific. Non-pest. Cue-lure.

Bactrocera endiandrae (Perkins \& May, 1949). Asia-Pacific. Non-pest. Methyl eugenol.

Bactrocera enochra (Drew, 1972). Asia-Pacific. Non-pest. Cue-lure.

Bactrocera epicharis (Hardy, 1970). Asia-Pacific. Non-pest. Cue-lure.

Bactrocera erubescentis (Drew \& Hancock, 1981). Asia-Pacific. Non-pest. Cue-lure.

Bactrocera eurycosta Drew \& Romig, 2013. Asia-Pacific. Non-pest. Cue-lure.

Bactrocera exigua (May, 1958). Asia-Pacific. Non-pest.

Bactrocera eximia Drew, 1989. Asia-Pacific. Non-pest.

Bactrocera expandens (Walker, 1859). Asia-Pacific. Fruit pest.

Bactrocera exspoliata (Hering, 1941). Asia-Pacific. Non-pest.

Bactrocera facialis (Coquillett, 1909). Asia-Pacific. Fruit pest. Cue-lure.

Bactrocera fagraea (Tryon, 1927). Asia-Pacific. Non-pest. Cue-lure.

Bactrocera fastigata Tsuruta \& White, 2001. Asia-Pacific. Non-pest. Cue-lure.

Bactrocera fergussoniensis Drew, 1989. Asia-Pacific. Non-pest.

Bactrocera fernandoi Tsuruta \& White, 2001. Asia-Pacific. Non-pest. Cue-lure.

Bactrocera finitima Drew, 1989. Asia-Pacific. Non-pest.

Bactrocera flavinotus (May, 1957). Asia-Pacific. Non-pest.

Bactrocera flavipennis (Hardy 1982). Asia-Pacific. Non-pest. Cue-lure.

Bactrocera flavoscutellata Lin \& Wang, 2005. Asia-Pacific. Non-pest. Cue-lure.

Notes: This is likely a junior synonym of $B$. pernigra. The only distinguishing character is in the width of the basal dark band on the scutellum, but this appears to be 
variable (Drew and Romig 2013). Because the characters have only been studied in small sample sizes there has not yet been an official synonymy.

Bactrocera flavosterna Drew \& Romig, 2013. Asia-Pacific. Non-pest. Cue-lure.

Bactrocera floresiae Drew \& Hancock, 1994. Asia-Pacific. Non-pest. Methyl eugenol. Bactrocera frauenfeldi (Schiner, 1868). Asia-Pacific. Fruit pest (polyphagous). Cue-lure, zingerone.

Notes: See under B. albistrigata.

Bactrocera froggatti (Bezzi, 1928). Asia-Pacific. Non-pest. Methyl eugenol. Bactrocera fuliginus (Drew \& Hancock, 1981). Asia-Pacific. Non-pest. Cue-lure. Bactrocera fulvicauda (Perkins, 1939). Asia-Pacific. Non-pest. Methyl eugenol. Bactrocera fulvifacies (Perkins, 1939). Asia-Pacific. Non-pest. Bactrocera fulvifemur Drew \& Hancock, 1994. Asia-Pacific. Non-pest. Cue-lure. Bactrocera fulvosterna Drew \& Romig, 2013. Asia-Pacific. Non-pest. Cue-lure. Bactrocera furcata David \& Hancock, 2017. Asia-Pacific. Non-pest. Bactrocera furfurosa Drew, 1989. Asia-Pacific. Non-pest. Cue-lure. Bactrocera furvescens Drew, 1989. Asia-Pacific. Non-pest. Cue-lure. Bactrocera furvilineata Drew, 1989. Asia-Pacific. Non-pest. Cue-lure. Bactrocera fuscalata Drew, 1989. Asia-Pacific. Non-pest. Methyl eugenol. Bactrocera fuscitibia Drew \& Hancock, 1994. Asia-Pacific. Non-pest. Cue-lure. Bactrocera fuscoformosa Drew \& Romig, 2013. Asia-Pacific. Non-pest. Cue-lure. Bactrocera fuscohumeralis White \& Evenhuis, 1999. Asia-Pacific. Non-pest. Bactrocera fuscolobata Drew \& Romig, 2013. Asia-Pacific. Non-pest. Cue-lure. Bactrocera fuscoptera Drew \& Romig, 2013. Asia-Pacific. Non-pest. Methyl eugenol. Bactrocera garciniae Bezzi, 1913. Asia-Pacific. Non-pest. Bactrocera gnetum Drew \& Hancock, 1995. Asia-Pacific. Non-pest. Bactrocera gombokensis Drew \& Hancock, 1994. Asia-Pacific. Non-pest. Cue-lure. Bactrocera grandifasciata White \& Evenhuis, 1999. Asia-Pacific. Non-pest. Bactrocera grandistylus Drew \& Hancock, 1995. Asia-Pacific. Non-pest. Bactrocera halfordiae (Tryon, 1927). Asia-Pacific. Fruit pest. Bactrocera halmaherae Drew \& Romig, 2013. Asia-Pacific. Non-pest. Cue-lure. Bactrocera hantanae Tsuruta \& White, 2001. Asia-Pacific. Non-pest. Cue-lure. Bactrocera harrietensis Ramani \& David, 2016. Asia-Pacific. Non-pest. Bactrocera hastigerina (Hardy, 1954). Asia-Pacific. Fruit pest (monophagous). Bactrocera hispidula (May, 1958). Asia-Pacific. Non-pest.

Bactrocera hollingsworthi Drew \& Romig, 2001. Asia-Pacific. Non-pest. Cue-lure. Bactrocera holtmanni (Hardy, 1974). Asia-Pacific. Non-pest. Cue-lure. Bactrocera humilis (Drew \& Hancock, 1981). Asia-Pacific. Non-pest. Bactrocera hyalina (Shiraki, 1933). Asia-Pacific. Non-pest. Bactrocera hypomelaina Drew, 1989. Asia-Pacific. Non-pest. Cue-lure. Bactrocera icelus (Hardy, 1974). Asia-Pacific. Non-pest.

Notes: We continue the use of a masculine epithet like in previous treatments. Hardy did not give an etymology in his description of the species, but 'icelus' could 
refer to the Greek mythical figure by that name, or reference to the Greek word for 'appearance', and we treat it as a noun in aposition.

Bactrocera illusioscutellaris Drew \& Romig, 2013. Asia-Pacific. Non-pest. Cue-lure, zingerone.

Notes: Zingerone is a new lure record.

Bactrocera impunctata (de Mejeire, 1914). Asia-Pacific. Non-pest. Methyl eugenol.

Bactrocera incompta Drew \& Romig, 2013. Asia-Pacific. Non-pest. Cue-lure.

Bactrocera inconspicua Drew \& Romig, 2013. Asia-Pacific. Non-pest. Methyl eugenol. Bactrocera inconstans Drew, 1989. Asia-Pacific. Non-pest. Cue-lure.

Bactrocera indecora (Drew 1971). Asia-Pacific. Non-pest. Cue-lure.

Bactrocera indonesiae Drew \& Hancock, 1994. Asia-Pacific. Non-pest. Methyl eugenol. Zingerone.

Bactrocera infulata Drew \& Hancock, 1994. Asia-Pacific. Non-pest. Methyl eugenol. Bactrocera invisitata Drew, 1989. Asia-Pacific. Non-pest. Methyl eugenol.

Bactrocera involuta (Hardy, 1982). Asia-Pacific. Non-pest. Cue-lure.

Bactrocera irvingiae Drew \& Hancock, 1994. Asia-Pacific. Non-pest.

Bactrocera ismayi Drew, 1989. Asia-Pacific. Non-pest. Methyl eugenol.

Notes: Methyl eugenol is a new lure record from surveys in Papua New Guinea in $1997 / 1999$.

Bactrocera jaceobancroftii Drew \& Romig, 2013. Asia-Pacific. Non-pest. Methyl eugenol. Bactrocera jarvisi (Tryon, 1927). Asia-Pacific. Fruit pest. Cue-lure, zingerone.

Bactrocera kalimantaniae Drew \& Romig, 2013. Asia-Pacific. Non-pest. Cue-lure.

Bactrocera kanchanaburi Drew \& Hancock , 1994. Asia-Pacific. Non-pest. Methyl eugenol. Bactrocera kandiensis Drew \& Hancock, 1994. Asia-Pacific. Fruit pest (polyphagous).

Methyl eugenol.

Notes: There is likely some (historical) introgression or hybridization between $B$.

kandiensis and $B$. dorsalis, and the two cannot be separated reliably using mitochondrial genes (Schutze et al. 2015a, 2015b, San Jose, unpublished data).

Bactrocera kelaena Drew, 1989. Asia-Pacific. Non-pest. Methyl eugenol.

Bactrocera kinabalu Drew \& Hancock, 1994. Asia-Pacific. Non-pest. Cue-lure.

Bactrocera kirki (Froggatt, 1910). Asia-Pacific. Fruit pest. Cue-lure.

Bactrocera kohkongiae Leblanc, 2015. Asia-Pacific. Non-pest. Cue-lure.

Bactrocera kraussi (Hardy, 1951). Asia-Pacific. Fruit pest. Cue-lure.

Bactrocera kuniyoshii (Shiraki, 1968). Asia-Pacific. Non-pest.

Bactrocera laithieniae Drew \& Romig, 2013. Asia-Pacific. Non-pest. Cue-lure.

Bactrocera lampabilis (Drew, 1971). Asia-Pacific. Non-pest. Methyl eugenol.

Bactrocera lata (Perkins 1938). Asia-Pacific. Non-pest. Cue-lure.

Bactrocera lateritaenia Drew \& Hancock, 1994. Asia-Pacific. Non-pest. Cue-lure.

Bactrocera laticaudus (Hardy, 1950). Asia-Pacific. Non-pest. Methyl eugenol.

Bactrocera laticosta Drew, 1989. Asia-Pacific. Non-pest. Cue-lure.

Bactrocera latifrons (Hendel, 1915). Asia-Pacific. Fruit pest (oligophagous).

Notes: Native to Asia and introduced into Africa and Hawaii. 
Bactrocera latilineata Drew, 1989. Asia-Pacific. Non-pest.

Notes: Male attractant uncertain, previous lure records are likely incorrect (see

Drew 1989).

Bactrocera latilineola Drew \& Hancock, 1994. Asia-Pacific. Non-pest. Methyl eugenol. Bactrocera latissima Drew, 1989. Asia-Pacific. Non-pest. Cue-lure.

Bactrocera limbifera (Bezzi, 1919). Asia-Pacific. Non-pest. Cue-lure.

Bactrocera linduensis Drew \& Romig, 2013. Asia-Pacific. Non-pest. Cue-lure.

Bactrocera lineata (Perkins, 1939). Asia-Pacific. Fruit pest (monophagous). Cue-lure. Bactrocera lombokensis Drew \& Hancock, 1994. Asia-Pacific. Non-pest. Cue-lure.

Bactrocera longicornis Macquart, 1835. Asia-Pacific. Non-pest. Cue-lure.

Notes: Type species for the genus (see Hardy 1976).

Bactrocera lucida (Munro, 1939). Africa. Non-pest.

Bactrocera luteola (Malloch, 1931). Asia-Pacific. Non-pest.

Bactrocera maculigera Doleschall, 1858. Asia-Pacific. Non-pest. Methyl eugenol.

Bactrocera makilingensis Drew \& Hancock, 1994. Asia-Pacific. Non-pest. Cue-lure.

Bactrocera malaysiensis Drew \& Hancock, 1994. Asia-Pacific. Non-pest. Cue-lure.

Bactrocera mamaliae Drew \& Romig, 2013. Asia-Pacific. Non-pest. Cue-lure.

Bactrocera manskii (Perkins \& May, 1949). Asia-Pacific. Non-pest. Cue-lure.

Bactrocera matsumurai (Shiraki, 1933). Asia-Pacific. Non-pest.

Bactrocera mayi (Hardy, 1951). Asia-Pacific. Non-pest. Methyl eugenol.

Bactrocera mcgregori (Bezzi, 1919). Asia-Pacific. Non-pest.

Bactrocera mediorufula Drew \& Romig, 2013. Asia-Pacific. Non-pest. Methyl eugenol. Bactrocera megaspilus (Hardy, 1982). Asia-Pacific. Non-pest. Cue-lure.

Bactrocera melania (Hardy \& Adachi, 1954). Asia-Pacific. Non-pest.

Bactrocera melanogaster Drew, 1989. Asia-Pacific. Non-pest. Methyl eugenol.

Bactrocera melanoscutata Drew, 1989. Asia-Pacific. Non-pest.

Bactrocera melanothoracica Drew, 1989. Asia-Pacific. Non-pest. Methyl eugenol.

Bactrocera melanotus (Coquillett, 1909). Asia-Pacific. Fruit pest. Cue-lure.

Bactrocera melas (Perkins \& May, 1949). Asia-Pacific. Fruit pest. Cue-lure.

Notes: It is uncertain if $B$. melas is a distinct species. Specimens identified as $B$.

melas may be a dark form of B. tryoni, or hybrids of $B$. tryoni and B. neohumeralis

(see Hancock et al. 2000).

Bactrocera melastomatos Drew \& Hancock, 1994. Asia-Pacific. Non-pest. Cue-lure.

Bactrocera memnonia (Drew, 1989). Asia-Pacific. Non-pest. Methyl eugenol.

Bactrocera menanus (Munro, 1984). Africa. Non-pest.

Bactrocera mendosa (May, 1958). Asia-Pacific. Non-pest.

Bactrocera merapiensis Drew \& Hancock, 1994. Asia-Pacific. Non-pest. Cue-lure.

Bactrocera mesomelas (Bezzi, 1908a). Africa. Fruit pest (monophagous).

Bactrocera mesonotaitha Drew, 1989. Asia-Pacific. Non-pest.

Bactrocera mesonotochra Drew, 1989. Asia-Pacific. Non-pest.

Bactrocera mimulus Drew, 1989. Asia-Pacific. Non-pest. Methyl eugenol.

Bactrocera minax (Enderlein, 1920). Asia-Pacific. Fruit pest.

Bactrocera minuscula Drew \& Hancock, 1994. Asia-Pacific. Non-pest. Methyl eugenol. 
Bactrocera minuta (Drew, 1971). Asia-Pacific. Non-pest. Cue-lure.

Bactrocera moluccensis (Perkins, 1939). Asia-Pacific. Fruit pest (monophagous). Cuelure, zingerone.

Bactrocera montyanus (Munro, 1984). Africa. Non-pest.

Bactrocera morobiensis Drew, 1989. Asia-Pacific. Non-pest. Cue-lure.

Bactrocera morula Drew, 1989. Asia-Pacific. Non-pest. Cue-lure.

Bactrocera mucronis (Drew, 1971). Asia-Pacific. Fruit pest. Cue-lure.

Bactrocera muiri (Hardy \& Adachi, 1954). Asia-Pacific. Non-pest.

Bactrocera munroi White, 2004. Africa. Non-pest.

Bactrocera murrayi (Perkins, 1939). Asia-Pacific. Fruit pest. Zingerone.

Bactrocera musae (Tryon, 1927). Asia-Pacific. Fruit pest (oligophagous). Methyl eugenol. Bactrocera mutabilis (May, 1952). Asia-Pacific. Fruit pest.

Bactrocera nanoarcuata Drew \& Romig, 2013. Asia-Pacific. Non-pest. Cue-lure.

Bactrocera nationigrotibialis Drew \& Romig, 2013. Asia-Pacific. Non-pest. Methyl eugenol. Bactrocera naucleae Drew \& Romig, 2001. Asia-Pacific. Non-pest. Methyl eugenol. Bactrocera neoarecae Drew, 2002. Asia-Pacific. Non-pest. Methyl eugenol. Bactrocera neocheesmanae Drew, 1989. Asia-Pacific. Non-pest. Methyl eugenol. Bactrocera neocognata Drew \& Hancock, 1994. Asia-Pacific. Non-pest. Cue-lure. Bactrocera neofulvicauda Drew \& Romig, 2013. Asia-Pacific. Non-pest. Cue-lure. Bactrocera neohumeralis (Hardy, 1951). Asia-Pacific. Fruit pest. Cue-lure, zingerone. Bactrocera neonigrita Drew, 1989. Asia-Pacific. Non-pest. Methyl eugenol. Bactrocera neonigrotibialis Drew, 2002. Asia-Pacific. Non-pest. Cue-lure. Bactrocera neopagdeni Drew, 1989. Asia-Pacific. Non-pest.

Bactrocera neopropinqua Drew \& Hancock, 1994. Asia-Pacific. Non-pest. Bactrocera neoritsemai Drew \& Romig, 2013. Asia-Pacific. Non-pest. Cue-lure. Bactrocera neoxanthodes Drew \& Romig, 2001. Asia-Pacific. Non-pest.

Bactrocera nesiotes (Munro, 1984). Africa. Non-pest.

Bactrocera nigella (Drew, 1968). Asia-Pacific. Non-pest. Methyl eugenol.

Bactrocera nigra (Tryon, 1927). Asia-Pacific. Non-pest.

Bactrocera nigrescens (Drew, 1968). Asia-Pacific. Non-pest. Methyl eugenol.

Bactrocera nigrescentis (Drew, 1971). Asia-Pacific. Non-pest. Cue-lure.

Bactrocera nigricula (Drew, 1989). Asia-Pacific. Non-pest.

Bactrocera nigrifacia Zhang Ji \& Chen, 2011. Asia-Pacific. Non-pest. Cue-lure.

Bactrocera nigrifemorata Li \& Wang, 2011. Asia-Pacific. Non-pest.

Bactrocera nigrita (Hardy, 1955). Asia-Pacific. Non-pest. Methyl eugenol.

Bactrocera nigrivenata (Munro, 1937). Africa. Non-pest.

Bactrocera nigrofemoralis White \& Tsuruta, 2001. Asia-Pacific. Non-pest. Cue-lure.

Bactrocera nigroscutata White \& Evenhuis, 1999. Asia-Pacific. Non-pest.

Bactrocera nigrotibialis (Perkins, 1938). Asia-Pacific. Fruit pest (oligophagous). Cue-lure.

Bactrocera nigrovittata Drew, 1989. Asia-Pacific. Non-pest.

Bactrocera notatagena (May, 1953). Asia-Pacific. Non-pest.

Bactrocera nothaphoebe Drew \& Romig, 2013. Asia-Pacific. Non-pest.

Bactrocera obfuscata Drew, 1989. Asia-Pacific. Non-pest. Cue-lure. 
Bactrocera oblineata Drew, 1989. Asia-Pacific. Non-pest. Cue-lure.

Bactrocera obliqua (Malloch, 1939). Asia-Pacific. Fruit pest.

Bactrocera obliquivenosa Drew \& Romig, 2001. Asia-Pacific. Non-pest. Methyl eugenol. Bactrocera obscura (Malloch, 1931). Asia-Pacific. Non-pest. Cue-lure.

Bactrocera obscurata (de Mejeire, 1911). Asia-Pacific. Non-pest.

Bactrocera obscurivitta Drew \& Romig, 2013. Asia-Pacific. Non-pest. Cue-lure.

Bactrocera obtrullata White \& Evenhuis, 1999. Asia-Pacific. Non-pest.

Bactrocera occipitalis (Bezzi, 1919). Asia-Pacific. Fruit pest. Methyl eugenol.

Notes: The pest status of this species is uncertain and has possibly been overrated in

literature, based on a few obscure rearing records cited in Drew and Hancock (1994).

Bactrocera ochracea Drew, 1989. Asia-Pacific. Non-pest. Cue-lure.

Bactrocera ochroma Drew \& Romig, 2013. Asia-Pacific. Fruit pest (monophagous).

Methyl eugenol.

Bactrocera ochromarginis (Drew, 1971). Asia-Pacific. Non-pest. Methyl eugenol.

Bactrocera ochrosiae (Malloch, 1942). Asia-Pacific. Non-pest. Cue-lure.

Bactrocera ochroventer Drew \& Romig, 2013. Asia-Pacific. Non-pest.

Notes: Male attractant uncertain. Label data of collected specimens suggests that

they have been collected both with cue lure and methyl eugenol, which seems

unlikely. Possibly the traps have been contaminated.

Bactrocera oleae (Gmelin, 1790). Africa. Fruit pest (monophagous).

Notes: Bactrocera oleae is thought to be native to sub-Saharan Africa, and invasive

in North Africa, southern Europe, western Asia, and California and northwestern

Mexico in North America.

Bactrocera opacovitta Drew \& Romig, 2013. Asia-Pacific. Non-pest. Methyl eugenol. Bactrocera opiliae (Drew \& Hardy, 1981). Asia-Pacific. Non-pest. Methyl eugenol. Bactrocera osbeckiae Drew \& Hancock, 1994. Asia-Pacific. Non-pest.

Bactrocera pacificae Drew \& Romig, 2001. Asia-Pacific. Non-pest.

Bactrocera pagdeni (Malloch, 1939). Asia-Pacific. Non-pest.

Bactrocera pallescentis (Hardy, 1955). Asia-Pacific. Non-pest.

Bactrocera pallida (Perkins \& May, 1949). Asia-Pacific. Non-pest. Methyl eugenol.

Bactrocera paraarecae Drew \& Romig, 2013. Asia-Pacific. Non-pest. Methyl eugenol.

Bactrocera parabancroftii Drew, 2011. Asia-Pacific. Non-pest. Cue-lure.

Bactrocera parabarringtoniae Drew \& Hancock, 1999. Asia-Pacific. Non-pest. Cue-lure. Bactrocera paradiospyri Chen Zhou \& Li, 2011. Asia-Pacific. Non-pest. Methyl eugenol. Bactrocera parafrauenfeldi Drew, 1989. Asia-Pacific. Non-pest. Cue-lure.

Bactrocera parafroggatti Drew \& Romig, 2001. Asia-Pacific. Non-pest. Methyl eugenol. Bactrocera paralatissima Drew \& Romig, 2013. Asia-Pacific. Non-pest. Cue-lure. Bactrocera paralimbifera Drew \& Romig, 2013. Asia-Pacific. Non-pest. Cue-lure. Bactrocera paramusae Drew, 1989. Asia-Pacific. Non-pest. Cue-lure.

Bactrocera paranigrita Drew \& Romi,g 2013. Asia-Pacific. Non-pest. Methyl eugenol. Bactrocera paraosbeckiae Drew, 2002. Asia-Pacific. Non-pest. Cue-lure.

Bactrocera paraverbascifoliae Drew, 2002. Asia-Pacific. Non-pest. Methyl eugenol. Bactrocera paraxanthodes Drew \& Hancock, 1995. Asia-Pacific. Non-pest. Methyl eugenol.

Notes: The attraction to methyl eugenol possibly is weak. 
Bactrocera parvula (Hendel, 1912). Asia-Pacific. Non-pest.

Bactrocera passiflorae (Froggatt, 1910). Asia-Pacific. Fruit pest. Cue-lure.

Bactrocera patula Drew \& Romig, 2013. Asia-Pacific. Non-pest. Cue-lure.

Bactrocera pectoralis (Walker, 1859). Asia-Pacific. Non-pest.

Bactrocera pedestris (Bezzi, 1913). Asia-Pacific. Non-pest. Cue-lure.

Bactrocera pendleburyi (Perkins, 1938). Asia-Pacific. Non-pest. Zingerone.

Notes: Zingerone is a new lure record.

Bactrocera peneallwoodi Drew \& Romig, 2013. Asia-Pacific. Non-pest.

Notes: Male attractant uncertain. Label data of collected specimens suggests that they have been collected both with cue lure and methyl eugenol, which seems unlikely. Possibly the traps have been contaminated.

Bactrocera penebeckerae Drew \& Romig, 2013. Asia-Pacific. Non-pest.

Bactrocera penecognata Drew \& Hancock, 1994. Asia-Pacific. Non-pest. Cue-lure.

Bactrocera penecorrecta Drew, 2002. Asia-Pacific. Non-pest. Methyl eugenol.

Bactrocera penecostalis Drew \& Romig, 2013. Asia-Pacific. Non-pest. Cue-lure.

Bactrocera penefurva Drew, 1989. Asia-Pacific. Non-pest.

Bactrocera peneobscura Drew \& Romig, 2001. Asia-Pacific. Non-pest. Cue-lure.

Bactrocera penephaea Drew \& Romig, 2013. Asia-Pacific. Non-pest. Cue-lure.

Bactrocera peninsularis (Drew \& Hancock, 1981). Asia-Pacific. Non-pest. Cue-lure.

Bactrocera pepisalae (Froggatt, 1910). Asia-Pacific. Non-pest. Methyl eugenol.

Bactrocera perfusca (Aubertin, 1929). Asia-Pacific. Fruit pest.

Bactrocera perigrapha White \& Tsuruta, 2001. Asia-Pacific. Non-pest. Cue-lure, zingerone.

Notes: Zingerone is a new lure record.

Bactrocera perkinsi (Drew \& Hancock, 1981). Asia-Pacific. Non-pest. Cue-lure.

Bactrocera pernigra Ito, 1983. Asia-Pacific. Non-pest. Cue-lure.

Notes: see comments under B. flavoscutellata

Bactrocera peterseni (Hardy, 1970). Asia-Pacific. Non-pest.

Bactrocera petila Drew, 1989. Asia-Pacific. Non-pest. Cue-lure.

Bactrocera phaea (Drew, 1971). Asia-Pacific. Non-pest. Cue-lure.

Bactrocera phaleriae (May, 1956). Asia-Pacific. Non-pest.

Bactrocera picea (Drew, 1972). Asia-Pacific. Non-pest. Methyl eugenol.

Bactrocera pictipennis Lin \& Zeng, 2011. Asia-Pacific. Non-pest. Methyl eugenol.

Bactrocera pisinna Drew, 1989. Asia-Pacific. Non-pest. Cue-lure.

Bactrocera popondettiensis Drew, 1989. Asia-Pacific. Non-pest.

Bactrocera profunda Tsuruta \& White, 2001. Asia-Pacific. Non-pest. Cue-lure.

Bactrocera prolixa Drew, 1989. Asia-Pacific. Non-pest. Methyl eugenol.

Bactrocera propedistincta Drew, 1989. Asia-Pacific. Non-pest.

Bactrocera propinqua (Hardy \& Adachi, 1954). Asia-Pacific. Non-pest. Cue-lure.

Bactrocera pruniae Drew \& Romig, 2013. Asia-Pacific. Fruit pest (monophagous).

Bactrocera pseudobeckerae Drew \& Romig, 2013. Asia-Pacific. Non-pest. Cue-lure.

Bactrocera pseudocucurbitae White, 1999, stat. rev. Asia-Pacific. Non-pest. Cue-lure.

Notes: This species was assigned to the subgenus Parasinodacus by Drew and Romig (2013), and subsequently assigned to genus Zeugodacus by De Meyer et al. (2015). It was assigned to Parasinodacus based on having a medial yellow scutal vitta and 
having just two scutellar setae, but it differs from other members of Parasinodacus in lacking yellow marks anterior to the transverse suture (= notopleural suture of Drew and Romig 2013), the presence of which is likely a reliable character for assignment to Zeugodacus (White 1999, San Jose et al. 2018). In a phylogeny based on molecular data from seven genes, the species is reliably placed within the Bactrocera clade (San Jose et al. 2018). We therefore here move the species back to Bactrocera and tentatively assign it to the subgenus Bactrocera.

Bactrocera pseudodistincta (Drew, 1971). Asia-Pacific. Non-pest. Cue-lure. Bactrocera pseudoversicolor Drew, 2002. Asia-Pacific. Non-pest. Methyl eugenol. Bactrocera psidii (Froggatt, 1899). Asia-Pacific. Fruit pest. Cue-lure. Bactrocera pulchra Tryon, 1927. Asia-Pacific. Non-pest. Bactrocera pusilla (Hardy, 1983). Asia-Pacific. Non-pest. Cue-lure. Bactrocera pyrifoliae Drew \& Hancock, 1994. Asia-Pacific. Fruit pest (oligophagous). Bactrocera quadrata (May, 1963). Asia-Pacific. Non-pest. Cue-lure. Bactrocera quadrisetosa (Bezzi, 1928). Asia-Pacific. Fruit pest. Bactrocera quasiinfulata Drew \& Romig, 2013. Asia-Pacific. Non-pest. Cue-lure. Bactrocera quasineonigrita Drew \& Romig, 2013. Asia-Pacific. Non-pest. Methyl eugenol. Bactrocera quasipropinqua Drew \& Hancock, 1994. Asia-Pacific. Non-pest.

Bactrocera quasisilvicola Drew, 1989. Asia-Pacific. Non-pest. Cue-lure. Bactrocera raiensis Drew \& Hancock, 1994. Asia-Pacific. Non-pest. Methyl eugenol. Bactrocera ramuensis Drew, 2011. Asia-Pacific. Non-pest. Cue-lure. Bactrocera ranganathi Drew \& Romig, 2013. Asia-Pacific. Non-pest. Methyl eugenol. Bactrocera reclinata Drew, 1989. Asia-Pacific. Non-pest. Methyl eugenol. Bactrocera recurrens (Hering, 1941). Asia-Pacific. Non-pest. Cue-lure. Bactrocera redunca (Drew, 1971). Asia-Pacific. Non-pest. Cue-lure. Bactrocera repanda Drew, 1989. Asia-Pacific. Non-pest.

Bactrocera resima (Drew, 1971). Asia-Pacific. Non-pest. Cue-lure. Bactrocera retrorsa Drew, 1989. Asia-Pacific. Non-pest. Methyl eugenol. Bactrocera rhabdota Drew, 1989. Asia-Pacific. Non-pest. Cue-lure. Bactrocera ritsemai (Weyenbergh, 1869). Asia-Pacific. Non-pest. Cue-lure.

Bactrocera robertsi Drew, 1989. Asia-Pacific. Non-pest. Cue-lure. Bactrocera robiginosa (May, 1958). Asia-Pacific. Non-pest.

Bactrocera romigae (Drew \& Hancock, 1981). Asia-Pacific. Non-pest. Methyl eugenol. Bactrocera rubigina (Wang \& Zhao, 1989). Asia-Pacific. Non-pest. Cue-lure, zingerone.

Notes: Zingerone is a new lure record.

Bactrocera rufescens (May, 1967). Asia-Pacific. Non-pest. Cue-lure.

Bactrocera rufivitta Drew, 2011. Asia-Pacific. Non-pest. Cue-lure.

Bactrocera rufofuscula (Drew \& Hancock, 1981). Asia-Pacific. Non-pest. Cue-lure, zingerone.

Bactrocera russeola (Drew \& Hancock, 1981). Asia-Pacific. Non-pest. Cue-lure. Bactrocera rutengiae Drew \& Romig, 2013. Asia-Pacific. Non-pest. Methyl eugenol. Bactrocera rutila (Hering, 1941). Asia-Pacific. Non-pest. Bactrocera samoae Drew, 1989. Asia-Pacific. Non-pest. 
Bactrocera sapaensis Drew \& Romig, 2013. Asia-Pacific. Non-pest. Cue-lure. Bactrocera satanellus (Hering, 1941). Asia-Pacific. Non-pest.

Bactrocera seguyi (Hering, 1939). Asia-Pacific. Non-pest. Methyl eugenol.

Bactrocera selenophora Tsuruta \& White, 2001. Asia-Pacific. Non-pest. Cue-lure. Bactrocera sembaliensis Drew \& Hancock, 1994. Asia-Pacific. Non-pest. Cue-lure.

Bactrocera setinervis (Malloch, 1938). Asia-Pacific. Non-pest.

Bactrocera silvicola (May, 1962). Asia-Pacific. Non-pest. Cue-lure, zingerone.

Bactrocera simulata (Malloch, 1939). Asia-Pacific. Non-pest. Cue-lure.

Bactrocera speculifer (Walker, 1865). Asia-Pacific. Fruit pest (monophagous). Methyl eugenol. Bactrocera speewahensis Fay \& Hancock, 2006. Asia-Pacific. Non-pest. Zingerone. Bactrocera splendida (Perkins, 1938). Asia-Pacific. Non-pest.

Bactrocera strigata (Perkins, 1934). Asia-Pacific. Non-pest.

Bactrocera sulawesiae Drew \& Hancock, 1994. Asia-Pacific. Non-pest. Methyl eugenol. Bactrocera suliae Drew \& Romig, 2013. Asia-Pacific. Non-pest. Methyl eugenol. Bactrocera sumbawaensis Drew \& Hancock, 1994. Asia-Pacific. Non-pest. Cue-lure. Bactrocera superba Drew \& Romig, 2013. Asia-Pacific. Non-pest. Cue-lure.

Bactrocera symplocos Drew \& Romig, 2013. Asia-Pacific. Non-pest.

Bactrocera syzygii White \& Tsuruta, 2001. Asia-Pacific. Non-pest. Zingerone.

Notes: Zingerone is a new lure record.

Bactrocera tapahensis Drew \& Romig, 2013. Asia-Pacific. Non-pest. Methyl eugenol. Bactrocera tenuifascia (May, 1965). Asia-Pacific. Non-pest. Methyl eugenol.

Bactrocera terminaliae Drew, 1989. Asia-Pacific. Non-pest.

Bactrocera terminifer (Walker, 1860). Asia-Pacific. Non-pest.

Bactrocera ternatiae Drew \& Romig, 2013. Asia-Pacific. Non-pest. Methyl eugenol. Bactrocera tetrachaeta (Bezzi, 1919). Asia-Pacific. Non-pest.

Bactrocera thailandica Drew \& Hancock, 1994. Asia-Pacific. Non-pest. Cue-lure.

Bactrocera thistletoni Drew, 1989. Asia-Pacific. Non-pest. Cue-lure.

Bactrocera tigrina (May, 1953). Asia-Pacific. Non-pest. Zingerone.

Bactrocera tillyardi (Perkins, 1938). Asia-Pacific. Non-pest.

Bactrocera tinomiscii Drew, 1989. Asia-Pacific. Non-pest. Cue-lure.

Bactrocera torresiae Huxam \& Hancock, 2006. Asia-Pacific. Non-pest. Cue-lure.

Bactrocera tortuosa White \& Evenhuis, 1999. Asia-Pacific. Non-pest.

Bactrocera toxopeusi (Hering, 1953). Asia-Pacific. Non-pest.

Bactrocera trifaria (Drew, 1971). Asia-Pacific. Non-pest. Cue-lure.

Bactrocera trifasciata (Hardy, 1982). Asia-Pacific. Non-pest. Cue-lure.

Bactrocera trilineola Drew, 1989. Asia-Pacific. Fruit pest. Cue-lure.

Bactrocera trivialis (Drew, 1971). Asia-Pacific. Fruit pest. Cue-lure, zingerone.

Bactrocera truncata Drew \& Romig, 2013. Asia-Pacific. Non-pest. Cue-lure.

Bactrocera tryoni (Froggatt, 1897). Asia-Pacific. Fruit pest. Cue-lure, zingerone.

Notes: See under B. aquilonis.

Bactrocera tsuneonis (Miyake, 1919). Asia-Pacific. Fruit pest.

Bactrocera tuberculata (Bezzi, 1916). Asia-Pacific. Fruit pest (polyphagous). Methyl eugenol. Bactrocera turneri Drew, 1989. Asia-Pacific. Non-pest. Cue-lure. 
Bactrocera umbrosa (Fabricius, 1805). Asia-Pacific. Fruit pest (monophagous). Methyl eugenol.

Bactrocera unifasciata (Malloch, 1939). Asia-Pacific. Non-pest. Cue-lure.

Bactrocera unilineata Drew, 1989. Asia-Pacific. Non-pest. Cue-lure.

Bactrocera unimacula Drew \& Hancock, 1994. Asia-Pacific. Non-pest. Methyl eugenol. Bactrocera unipunctata (Malloch, 1939). Asia-Pacific. Non-pest.

Bactrocera unistriata (Drew, 1971). Asia-Pacific. Non-pest. Methyl eugenol.

Bactrocera unitaeniola Drew \& Romig, 2001. Asia-Pacific. Non-pest. Cue-lure.

Bactrocera usitata Drew \& Hancock, 1994. Asia-Pacific. Non-pest. Cue-lure.

Bactrocera ustulata Drew, 1989. Asia-Pacific. Non-pest. Cue-lure.

Bactrocera uvariae Drew, 2011. Asia-Pacific. Non-pest. Cue-lure.

Bactrocera venefica (Hering, 1938). Asia-Pacific. Non-pest.

Bactrocera verbascifoliae Drew \& Hancock, 1994. Asia-Pacific. Non-pest. Methyl eugenol. Bactrocera versicolor (Bezzi, 1916). Asia-Pacific. Fruit pest (monophagous). Methyl eugenol. Bactrocera visenda (Hardy, 1951). Asia-Pacific. Non-pest. Methyl eugenol. Bactrocera vishnu Drew \& Hancock, 1994. Asia-Pacific. Non-pest. Cue-lure. Bactrocera vulgaris (Drew, 1971). Asia-Pacific. Non-pest. Cue-lure.

Bactrocera waaiae Drew \& Romig, 2013. Asia-Pacific. Non-pest. Methyl eugenol.

Bactrocera wanangiae Drew \& Hancock, 2016. Asia-Pacific. Non-pest.

Bactrocera warisensis White \& Evenhuis, 1999. Asia-Pacific. Non-pest.

Bactrocera wuzhishana Li \& Wang, 2006. Asia-Pacific. Non-pest. Methyl eugenol.

Bactrocera xanthodes (Broun, 1904). Asia-Pacific. Fruit pest. Methyl eugenol.

Bactrocera yayeyamana (Matsumara, 1916). Asia-Pacific. Non-pest.

Bactrocera yorkensis Drew \& Hancock, 1999. Asia-Pacific. Non-pest. Methyl eugenol. Bactrocera zonata (Saunders, 1842). Asia-Pacific. Fruit pest (polyphagous). Methyl eugenol.

Notes: Natively distributed in Asia, from the Indian subcontinent to Vietnam, and invasive in the Afrotropical and West-Palearctic (Middle East) Regions.

\section{Genus Dacus Fabricius}

Dacus abbabae Munro, 1933. Africa. Non-pest.

Dacus abditus (Munro, 1984). Africa. Non-pest.

Dacus abruptus White, 2009. Africa. Non-pest.

Dacus absonifacies (May, 1956). Asia-Pacific. Non-pest. Cue-lure, zingerone.

Dacus acutus White, 2009. Africa. Non-pest.

Dacus adenae (Hering, 1940). Africa. Non-pest.

Dacus adenionis Munro, 1984. Africa. Non-pest.

Dacus adustus Munro, 1948. Africa. Non-pest.

Dacus aequalis Coquillett, 1909. Asia-Pacific. Non-pest. Cue-lure, zingerone.

Dacus africanus Adams, 1905. Africa. Non-pest. Cue-lure.

Dacus alarifumidus Drew, 1989. Asia-Pacific. Non-pest. Cue-lure.

Dacus albiseta White, 2009. Africa. Non-pest. Cue-lure.

Dacus alulapictus Drew, 1989. Asia-Pacific. Non-pest.

Dacus amberiens (Munro, 1984). Africa. Non-pest.

Dacus ambonensis Drew \& Hancock, 1998. Asia-Pacific. Non-pest. Cue-lure. 
Dacus amphoratus (Munro, 1984). Africa. Non-pest.

Dacus aneuvittatus (Drew, 1971). Asia-Pacific. Non-pest.

Dacus annulatus Becker, 1903. Africa. Non-pest.

Dacus apectus White, 2006. Africa. Non-pest.

Dacus apiculatus White, 2006. Africa. Non-pest. Cue-lure.

Dacus apostata (Hering, 1937). Africa. Non-pest.

Dacus apoxanthus Bezzi, 1924. Africa. Non-pest.

Dacus arabicus White, 2006. Africa. Non-pest.

Dacus arcuatus Munro, 1939. Africa. Non-pest.

Dacus armatus Fabricius, 1805. Africa. Non-pest. Cue-lure.

Notes: Type species for the genus.

Dacus aspilus Bezzi, 1924. Africa. Non-pest.

Dacus atrimarginatus Drew \& Hancock, 1998. Asia-Pacific. Non-pest.

Dacus attenuatus Collart, 1935. Africa. Non-pest.

Dacus axanthinus White \& Evenhuis, 1999. Asia-Pacific. Non-pest.

Dacus axanus (Hering 1938). Asia-Pacific. Cucurbitaceae fruit pest. Cue-lure, zingerone.

Notes: Dacus axanus is a species that is common in Australia and Papua New

Guinea, and this name appears in various pest-related resources. D. unicolor and

$D$. vespiformis may be conspecific with $D$. axanus. The latter two were described

in a single publication by Hendel (Hendel 1927) without illustrations and the descriptions do not differentiate either from $D$. axanus. The types of $D$. unicolor and D. vespiformis were lost during the fire at the Museum in Hamburg in 1943.

Dacus badius Drew, 1989. Asia-Pacific. Non-pest. Cue-lure.

Dacus bakingiliensis Hancock, 1985. Africa. Non-pest. Cue-lure.

Dacus bannatus Wang, 1990. Asia-Pacific. Non-pest. Cue-lure.

Dacus basifasciatus (Hering, 1941). Africa. Non-pest.

Dacus bellulus Drew \& Hancock, 1981. Asia-Pacific. Non-pest. Cue-lure, zingerone.

Dacus bequaerti Collart, 1935. Africa. Non-pest.

Dacus bidens (Curran, 1927). Africa. Non-pest.

Dacus binotatus Loew, 1862. Africa. Non-pest. Cue-lure.

Dacus bispinosus (Wang, 1990). Asia-Pacific. Non-pest.

Dacus bistrigulatus Bezzi, 1908. Africa. Non-pest.

Dacus bivittatus (Bigot, 1858). Africa. Cucurbitaceae fruit pest. Cue-lure.

Dacus blepharogaster Bezzi, 1917. Africa. Non-pest.

Dacus bombastus Hering, 1941. Africa. Non-pest.

Dacus botianus (Munro, 1984). Africa. Non-pest.

Dacus brevis Coquillett, 1901. Africa. Non-pest.

Dacus brevistriga Walker, 1861. Africa. Non-pest.

Dacus briani White, 2006. Africa. Non-pest.

Dacus brunnalis White, 2009. Africa. Non-pest.

Dacus calirayae Drew \& Hancock, 1998. Asia-Pacific. Non-pest. Cue-lure.

Dacus capillaris (Drew, 1972). Asia-Pacific. Non-pest. Cue-lure.

Dacus carnesi (Munro, 1984). Africa. Non-pest. 
Dacus carvalhoi (Munro, 1984). Africa. Non-pest.

Dacus ceropegiae (Munro, 1984). Africa. Non-pest.

Dacus chamun (Munro, 1984). Africa. Non-pest.

Dacus chapini Curran, 1927. Africa. Non-pest.

Dacus chiwira Hancock, 1985. Africa. Non-pest. Cue-lure.

Dacus chrysomphalus (Bezzi, 1924). Africa. Non-pest. Cue-lure.

Dacus ciliatus Loew, 1862 Africa. Cucurbitaceae fruit pest.

Notes: Native to the Aftrotropical Region, and invasive in the Middle East and the

Indian subcontinent (India, Pakistan, Bangladesh, Sri Lanka).

Dacus clinophlebs Hendel, 1928. Africa. Non-pest.

Dacus coenensis Royer \& Hancock, 2012. Asia-Pacific. Non-pest. Cue-lure.

Dacus collarti Munro, 1938. Africa. Non-pest.

Dacus congoensis White, 2006. Africa. Non-pest.

Dacus conopsoides de Meijere, 1911. Asia-Pacific. Non-pest.

Dacus copelandi White, 2006. Africa. Non-pest.

Dacus crabroniformis (Bezzi, 1914). Asia-Pacific. Non-pest.

Dacus croceus Munro, 1957. Africa. Non-pest.

Dacus cyathus (Munro, 1984). Africa. Non-pest.

Dacus delicatus Munro, 1939. Africa. Non-pest.

Dacus deltatus White, 2006. Africa. Non-pest.

Dacus demmerezi (Bezzi, 1917). Africa. Cucurbitaceae fruit pest. Cue-lure.

Dacus devure Hancock, 1985. Africa. Non-pest. Cue-lure.

Dacus diastatus Munro, 1984. Africa. Non-pest. Cue-lure.

Dacus discipennis (Walker, 1861). Asia-Pacific. Non-pest.

Dacus discophorus (Hering, 1956). Asia-Pacific. Non-pest. Cue-lure.

Dacus discors Drew, 1989. Asia-Pacific. Non-pest. Cue-lure.

Dacus discretus Drew \& Romig, 2013. Asia-Pacific. Non-pest. Cue-lure.

Dacus disjunctus (Bezzi, 1915). Africa. Non-pest.

Dacus dissimilis Drew, 1989. Asia-Pacific. Non-pest. Cue-lure.

Dacus donggaliae Drew \& Romig, 2013. Asia-Pacific. Non-pest. Cue-lure.

Dacus dorjii Drew \& Romig, 2007. Asia-Pacific. Non-pest. Cue-lure.

Dacus durbanensis Munro, 1935. Africa. Non-pest. Cue-lure, zingerone.

Notes: The attraction to zingerone was documented by Manrakhan et al. (2017).

Dacus eclipsis (Bezzi, 1924). Africa. Non-pest. Cue-lure.

Dacus elatus White, 2006. Africa. Non-pest.

Dacus elegans (Munro, 1984). Africa. Non-pest.

Dacus elutissimus Bezzi, 1924. Africa. Non-pest.

Dacus eminus Munro 1939. Africa. Non-pest. Cue-lure.

Dacus erythraeus Bezzi, 1917. Africa. Non-pest.

Dacus esakii (Shiraki, 1939). Asia-Pacific. Non-pest.

Dacus etiennellus Munro, 1984. Africa. Non-pest. Cue-lure.

Dacus externellus (Munro, 1984). Africa. Non-pest.

Dacus famona Hancock, 1985. Africa. Non-pest. Cue-lure. 
Dacus fasciolatus Collart, 1940. Africa. Non-pest.

Dacus feijeni White, 1998. Asia-Pacific. Non-pest. Cue-lure.

Dacus ficicola Bezzi, 1915. Africa. Non-pest.

Dacus fissuratus White, 2006. Africa. Non-pest.

Dacus flavicrus Graham, 1910. Africa. Non-pest.

Dacus fletcheri Drew \& Romig, 2007. Asia-Pacific. Non-pest. Cue-lure.

Dacus formosanus (Tseng \& Chu, 1983). Asia-Pacific. Non-pest. Cue-lure.

Dacus freidbergi (Munro, 1984). Africa. Non-pest.

Dacus frontalis Becker, 1922. Africa. Cucurbitaceae fruit pest. Cue-lure, zingerone.

Notes: The attraction to zingerone was documented by Manrakhan et al. (2017).

Dacus fumosus Collart, 1935. Africa. Non-pest.

Dacus fuscatus Wiedemann, 1819. Africa. Non-pest.

Dacus fuscinervis Malloch, 1932. Africa. Non-pest.

Dacus fuscovittatus Graham, 1910. Africa. Non-pest. Cue-lure.

Dacus gabonensis White, 2006. Africa. Non-pest.

Dacus ghesquierei Collart, 1935. Africa. Non-pest.

Dacus goergeni De Meyer, White \& Goodger, 2013. Africa. Non-pest.

Dacus guineensis Hering, 1944. Africa. Non-pest.

Dacus gypsoides Munro, 1933. Africa. Non-pest.

Dacus hainanus Wang \& Zhao, 1989. Asia-Pacific. Non-pest.

Dacus hamatus Bezzi, 1917. Africa. Non-pest.

Dacus hapalus (Munro, 1984). Africa. Non-pest.

Dacus hardyi Drew, 1979. Asia-Pacific. Non-pest. Cue-lure.

Dacus hargreavesi (Munro, 1939). Africa. Non-pest.

Dacus herensis (Munro, 1984). Africa. Non-pest.

Dacus humeralis (Bezzi, 1915). Africa. Non-pest. Cue-lure.

Dacus hyalobasis Bezzi, 1924. Africa. Non-pest.

Dacus iaspideus Munro, 1948. Africa. Non-pest.

Dacus icariiformis (Enderlein, 1920). Asia-Pacific. Non-pest.

Dacus ikelenge Hancock, 1985. Africa. Non-pest. Cue-lure.

Dacus impar Drew, 1989. Asia-Pacific. Non-pest.

Dacus inclytus (Munro, 1984). Africa. Non-pest.

Dacus indecorus (Hardy, 1974). Asia-Pacific. Non-pest.

Dacus infernus (Hardy, 1973). Asia-Pacific. Non-pest.

Dacus inflatus Munro, 1939. Africa. Non-pest.

Dacus inornatus Bezzi, 1908. Africa. Non-pest.

Dacus insolitus White, 2009. Africa. Non-pest.

Dacus insulosus Drew \& Hancock, 1998. Asia-Pacific. Non-pest.

Dacus jubatus (Munro, 1984). Africa. Non-pest.

Dacus kakamega White, 2006. Africa. Non-pest.

Dacus kaplanae White, 2009. Africa. Non-pest.

Dacus kariba Hancock, 1985. Africa. Non-pest. Cue-lure.

Dacus katonae Bezzi, 1924. Africa. Non-pest. 
Dacus keiseri (Hering, 1956). Asia-Pacific. Non-pest.

Dacus kurrensis White, 2009. Africa. Non-pest.

Dacus lagunae Drew \& Hancock, 1998. Asia-Pacific. Non-pest. Cue-lure.

Dacus langi Curran, 1927. Africa. Non-pest. Cue-lure.

Dacus leongi Drew \& Hancock, 1998. Asia-Pacific. Non-pest. Cue-lure.

Dacus limbipennis Macquart, 1843. Africa. Cucurbitaceae fruit pest.

Dacus linearis Collart, 1935. Africa. Non-pest.

Dacus longicornis (Wiedemann, 1830). Asia-Pacific. Cucurbitaceae fruit pest. Cue-lure.

Dacus longistylus Wiedemann, 1830. Africa. Non-pest.

Dacus lotus (Bezzi, 1924). Africa. Non-pest.

Dacus lounsburyii Coquillett, 1901. Africa. Cucurbitaceae fruit pest.

Dacus luteovittatus White, 2009. Africa. Non-pest.

Dacus macer Bezzi, 1919. Africa. Non-pest.

Dacus maculipterus Drew \& Hancock, 1998. Asia-Pacific. Non-pest.

Dacus madagascarensis White, 2006. Africa. Non-pest. Cue-lure.

Dacus magnificus White, 2009. Africa. Non-pest.

Dacus maprikensis Drew, 1989. Asia-Pacific. Non-pest.

Dacus marshalli Bezzi, 1924. Africa. Non-pest.

Dacus masaicus Munro, 1937. Africa. Non-pest. Cue-lure.

Dacus mayi (Drew, 1972). Asia-Pacific. Non-pest. Cue-lure.

Dacus maynei Bezzi, 1924. Africa. Non-pest.

Dacus mediovittatus White, 2006. Africa. Non-pest. Cue-lure.

Dacus meladassus (Munro, 1984). Africa. Non-pest.

Dacus melanaspis (Munro, 1984). Africa. Non-pest.

Dacus melanohumeralis Drew, 1989. Asia-Pacific. Non-pest. Methyl eugenol.

Dacus melanopectus Drew \& Romig, 2013. Asia-Pacific. Non-pest. Methyl eugenol.

Dacus merzi White, 2006. Africa. Non-pest.

Dacus mirificus (Munro, 1984). Africa. Non-pest.

Dacus mochii Bezzi, 1917. Africa. Non-pest.

Dacus mulgens Munro, 1932. Africa. Non-pest.

Dacus murphyi Drew \& Hancock, 1998. Asia-Pacific. Non-pest.

Dacus nairobensis White, 2006. Africa. Non-pest.

Dacus namibiensis Hancock \& Drew, 2001. Africa. Non-pest.

Dacus nanggalae Drew \& Hancock, 1998. Asia-Pacific. Non-pest. Cue-lure.

Dacus nanus Collart, 1940. Africa. Non-pest.

Dacus newmani (Perkins, 1937). Asia-Pacific. Non-pest. Cue-lure.

Dacus nigriscutatus White, 2006. Africa. Non-pest.

Dacus nigrolateris White, 2006. Africa. Non-pest.

Dacus notalaxus Munro, 1984. Africa. Non-pest.

Dacus nummularius (Bezzi, 1916). Asia-Pacific. Non-pest. Cue-lure.

Dacus obesus Munro, 1948. Africa. Non-pest.

Dacus okumuae White, 2006. Africa. Non-pest.

Dacus ooii Drew \& Hancock, 1998. Asia-Pacific. Non-pest. Cue-lure. 
Dacus opacatus Munro, 1948. Africa. Non-pest.

Dacus ortholomatus Hardy, 1982. Asia-Pacific. Non-pest.

Dacus ostiofaciens Munro, 1932. Africa. Non-pest.

Dacus pallidilatus Munro, 1948. Africa. Non-pest. Cue-lure.

Dacus palmerensis Drew, 1989. Asia-Pacific. Non-pest. Cue-lure.

Dacus pamelae (Munro, 1984). Africa. Non-pest.

Dacus panpyrrhus (Munro, 1984). Africa. Non-pest.

Dacus parvimaculatus White, 2006. Africa. Non-pest.

Dacus pecropsis Munro, 1984. Africa. Non-pest. Cue-lure.

Dacus pedunculatus (Bezzi, 1919). Asia-Pacific. Non-pest.

Dacus pergulariae Munro, 1938. Africa. Non-pest.

Dacus persicus Hendel, 1927. Asia-Pacific. Non-pest.

Dacus petioliforma (May, 1956). Asia-Pacific. Non-pest. Cue-lure.

Dacus phantoma Hering, 1941. Africa. Non-pest.

Dacus phimis (Munro, 1984). Africa. Non-pest.

Dacus phloginus (Munro, 1984). Africa. Non-pest.

Dacus pictus (Hardy, 1970). Asia-Pacific. Non-pest.

Dacus plagiatus Collart, 1935. Africa. Non-pest.

Dacus pleuralis Collart, 1935. Africa. Non-pest. Cue-lure.

Dacus polistiformis (Senior-White, 1922). Asia-Pacific. Non-pest.

Dacus pseudapostata White, 2009. Africa. Non-pest.

Dacus pseudomirificus White, 2009. Africa. Non-pest.

Dacus pulchralis White, 2006. Africa. Non-pest. Cue-lure.

Dacus pullescens Munro, 1948. Africa. Non-pest.

Dacus pullus (Hardy, 1982). Asia-Pacific. Non-pest.

Dacus punctatifrons Karsch, 1887. Africa. Cucurbitaceae fruit pest. Cue-lure.

Dacus purpurifrons Bezzi, 1924. Africa. Non-pest.

Dacus purus (Curran, 1927). Africa. Non-pest.

Dacus pusillator (Munro, 1984). Africa. Non-pest.

Dacus pusillus (May, 1965). Asia-Pacific. Non-pest. Methyl eugenol.

Dacus quilicii White, 2006. Africa. Non-pest. Cue-lure.

Dacus radmirus Hering, 1941. Africa. Non-pest.

Dacus ramanii Drew \& Hancock, 1998. Asia-Pacific. Non-pest. Cue-lure.

Dacus rubicundus Bezzi, 1924. Africa. Non-pest.

Dacus rufoscutellatus (Hering, 1937). Africa. Non-pest.

Dacus rufus Bezzi, 1915. Africa. Non-pest.

Dacus rugatus Munro, 1984. Africa. Non-pest.

Dacus ruslan (Hering, 1941). Africa. Non-pest.

Dacus rutilus Munro, 1948. Africa. Non-pest.

Dacus sakeji Hancock, 1985. Africa. Non-pest. Cue-lure.

Dacus salamander (Drew \& Hancock, 1981). Asia-Pacific. Non-pest. Cue-lure.

Dacus santongae Drew \& Hancock, 1998. Asia-Pacific. Non-pest. Cue-lure.

Dacus satanas (Hering, 1939). Asia-Pacific. Non-pest. Zingerone. 
Notes: Zingerone is a new lure record. Dacus scaber Loew, 1862. Africa. Non-pest. Dacus schoutedeni Collart, 1935. Africa. Non-pest.

Dacus secamoneae Drew, 1989. Asia-Pacific. Non-pest. Cue-lure, zingerone.

Dacus segunii White, 2006. Africa. Non-pest. Cue-lure.

Dacus seguyi (Munro, 1984). Africa. Non-pest.

Dacus semisphaereus Becker, 1903. Africa. Non-pest.

Dacus senegalensis White, 2009. Africa. Non-pest.

Dacus serratus (Munro, 1984). Africa. Non-pest.

Dacus setilatens Munro, 1984. Africa. Non-pest.

Dacus siamensis Drew \& Hancock, 1998. Asia-Pacific. Non-pest. Cue-lure.

Dacus signatifrons (May, 1956). Asia-Pacific. Non-pest. Cue-lure.

Dacus siliqualactis Munro, 1939. Africa. Non-pest.

Dacus sinensis Wang, 1990. Asia-Pacific. Non-pest.

Dacus solomonensis Malloch, 1939. Asia-Pacific. Cucurbitaceae fruit pest. Cue-lure.

Dacus sphaeristicus Speiser, 1910. Africa. Non-pest.

Dacus sphaeroidalis (Bezzi, 1916). Asia-Pacific. Non-pest. Cue-lure.

Dacus sphaerostigma (Bezzi, 1924). Africa. Non-pest.

Dacus spissus Munro, 1984. Africa. Non-pest.

Dacus stentor Munro, 1929. Africa. Non-pest.

Dacus stylifer (Bezzi, 1919). Africa. Non-pest.

Dacus subsessilis (Bezzi, 1919). Asia-Pacific. Non-pest.

Dacus succaelestis Ito, 2011. Asia-Pacific. Non-pest.

Dacus taui Drew \& Romig, 2001. Asia-Pacific. Non-pest. Cue-lure.

Dacus telfaireae (Bezzi, 1924). Africa. Non-pest. Cue-lure.

Dacus temnopterus Bezzi, 1928. Africa. Non-pest.

Dacus tenebricus Munro, 1938. Africa. Non-pest.

Dacus tenebrosus Drew \& Hancock, 1998. Asia-Pacific. Non-pest. Cue-lure, zingerone.

Notes: Zingerone is a new lure record.

Dacus theophrastus Hering, 1941. Africa. Non-pest. Cue-lure.

Dacus transitorius Collart, 1935. Africa. Non-pest.

Dacus transversalis White, 2009. Africa. Non-pest.

Dacus triater Munro, 1937. Africa. Non-pest.

Dacus trigonus Bezzi, 1919. Africa. Non-pest.

Dacus trimacula Wang, 1990. Asia-Pacific. Non-pest. Cue-lure, zingerone.

Notes: Zingerone is a new lure record.

Dacus triquetrus Drew \& Romig, 2013. Asia-Pacific. Non-pest. Cue-lure.

Dacus umbeluzinus (Munro, 1984). Africa. Non-pest.

Dacus umbrilatus Munro, 1938. Africa. Non-pest.

Dacus umehi White, 2006. Africa. Non-pest.

Dacus unicolor (Hendel, 1927). Asia-Pacific. Non-pest.

Notes: See under D. axanus

Dacus velutifrons White, 2009. Africa. Non-pest. 
Dacus venetatus Munro, 1939. Africa. Non-pest. Cue-lure.

Dacus vertebratus Bezzi, 1908. Africa. Cucurbitaceae fruit pest. Cue-lure.

Dacus vespiformis (Hendel, 1927). Asia-Pacific. Non-pest.

Notes: See under D. axanus.

Dacus vestigivittatus White, 2009. Africa. Non-pest.

Dacus viator Munro, 1939. Africa. Non-pest.

Dacus vijaysegarani Drew \& Hancock, 1998. Asia-Pacific. Non-pest. Cue-lure, zingerone.

Notes: Zingerone is a new lure record.

Dacus vittatus (Hardy, 1974). Asia-Pacific. Non-pest.

Dacus wallacei White, 1998. Asia-Pacific. Non-pest.

Dacus woodi Bezzi, 1917. Africa. Non-pest.

Dacus xanthaspis (Munro, 1984). Africa. Non-pest.

Dacus xanthinus White, 2009. Africa. Non-pest.

Dacus xanthopterus (Bezzi, 1915). Africa. Non-pest. Cue-lure.

Dacus xanthopus Bezzi, 1924. Africa. Non-pest.

Dacus yangambinus Munro, 1984. Africa. Non-pest.

Dacus yaromi White, 2009. Africa. Non-pest.

Dacus yemenensis White, 2006. Africa. Non-pest.

\section{Genus Monacrostichus Bezzi}

Monacrostichus citricola (Bezzi, 1913). Asia-Pacific. Fruit pest.

Notes: Type species for the genus.

Monacrostichus malaysiae Drew \& Hancock, 1994. Asia-Pacific. Non-pest.

\section{Genus Zeugodacus Hendel}

Zeugodacus abdoangustus (Drew, 1972). Asia-Pacific. Non-pest. Cue-lure.

Zeugodacus abdoaurantiacus (Drew, 1989). Asia-Pacific. Non-pest.

Zeugodacus abdopallescens (Drew, 1971). Asia-Pacific. Non-pest. Cue-lure.

Zeugodacus ablepharus (Bezzi, 1919). Asia-Pacific. Non-pest.

Zeugodacus abnormis (Hardy, 1982). Asia-Pacific. Non-pest. Cue-lure.

Zeugodacus absolutus (Walker, 1861). Asia-Pacific. Non-pest.

Zeugodacus aithonota (Drew \& Romig, 2013). Asia-Pacific. Non-pest. Cue-lure.

Zeugodacus alampetus (Drew, 1989). Asia-Pacific. Non-pest. Methyl eugenol.

Zeugodacus ambiguus (Shiraki, 1933). Asia-Pacific. Non-pest. Cue-lure.

Zeugodacus amoenus (Drew, 1972). Asia-Pacific. Non-pest. Cue-lure.

Zeugodacus anala (Chen \& Zhou, 2013). Asia-Pacific. Non-pest.

Notes: We regard this name as a noun, not changing the ending, following De

Meyer et al. (2015). Chen and Zhou (2013) did not specify if it was meant as a noun or adjective, but mentioned "the specific ephithet refers to the wing anal streak".

Zeugodacus anchitrichotus (Drew, 1989). Asia-Pacific. Non-pest.

Zeugodacus angusticostatus (Drew, 1989). Asia-Pacific. Non-pest. Cue-lure.

Zeugodacus angustifinis (Hardy, 1982). Asia-Pacific. Non-pest. Cue-lure.

Zeugodacus apicalis (de Meijere, 1911). Asia-Pacific. Non-pest. Cue-lure.

Zeugodacus apiciflavus (Yu He \& Chen, 2011). Asia-Pacific. Non-pest. 
Zeugodacus apicofemoralis (Drew \& Romig, 2013). Asia-Pacific. Non-pest. Cue-lure. Zeugodacus areolatus (Walker, 1861). Asia-Pacific. Non-pest.

Zengodacus arisanicus Shiraki, 1933, stat. rev. Asia-Pacific. Non-pest. Cue-lure.

Notes: This species is here reassigned to Zeugodacus. It has a medial postsutural vitta and yellow markings anterior of the transverse suture, which are likely reliable morphological characters for assignment to Zeugodacus. This generic assignment is further supported by DNA sequence data from seven genes (San Jose et al. 2018). Whether the other members assigned to the subgenus Hemizengodacus should be placed in Bactrocera or Zeugodacus remains to be determined.

Zeugodacus armillatus (Hering, 1938). Asia-Pacific. Non-pest.

Zeugodacus assamensis White, 1999. Asia-Pacific. Non-pest. Cue-lure.

Zeugodacus atrichus (Bezzi, 1919). Asia-Pacific. Non-pest.

Zeugodacus atrifacies (Perkins, 1938). Asia-Pacific. Non-pest. Cue-lure.

Zeugodacus atrisetosus (Perkins, 1939). Asia-Pacific. Cucurbitaceae fruit pest.

Zeugodacus atypicus (White \& Evenhuis, 1999). Asia-Pacific. Non-pest.

Zeugodacus aurantiventer (Drew, 1989). Asia-Pacific. Non-pest. Cue-lure.

Zeugodacus bakeri (Bezzi, 1919). Asia-Pacific. Non-pest.

Zeugodacus baliensis (Drew \& Romig, 2013). Asia-Pacific. Non-pest. Cue-lure.

Zeugodacus baoshanensis (Zhang, Ji, Yang \& Chen, 2011). Asia-Pacific. Non-pest.

Zeugodacus biguttatus (Bezzi, 1916). Asia-Pacific. Non-pest. Cue-lure.

Zengodacus binoyi (Drew, 2002). Asia-Pacific. Non-pest. Cue-lure.

Zeugodacus bogorensis (Hardy, 1983). Asia-Pacific. Non-pest. Cue-lure.

Zeugodacus borongensis (Drew \& Romig, 2013). Asia-Pacific. Non-pest. Cue-lure.

Zeugodacus brachus (Drew, 1972). Asia-Pacific. Non-pest. Cue-lure.

Zeugodacus brevipunctatus (David \& Hancock, 2017), comb. n. Asia-Pacific. Non-pest.

Cue-lure.

Notes: This species was recently described in Bactrocera and placed in the subgenus

Sinodacus, of which all other previous members have been transferred to Zeugoda-

cus (De Meyer et al. 2015). We here follow this reasoning.

Zeugodacus brevivitta (Drew \& Romig, 2013). Asia-Pacific. Non-pest.

Zeugodacus buruensis (White, 1999). Asia-Pacific. Non-pest. Cue-lure.

Zeugodacus buvittatus (Drew, 1989). Asia-Pacific. Non-pest. Cue-lure.

Zeugodacus calumniatus (Hardy, 1970). Asia-Pacific. Non-pest. Methyl eugenol.

Zeugodacus careomacula (Drew \& Romig, 2013). Asia-Pacific. Non-pest. Cue-lure.

Zeugodacus caudatus (Fabricius, 1805). Asia-Pacific. Cucurbitaceae flower pest. Cue-lure.

Notes: Type species for genus.

Zeugodacus choristus (May, 1962). Asia-Pacific. Non-pest. Cue-lure.

Zeugodacus cilifer (Hendel, 1912). Asia-Pacific. Non-pest. Cue-lure.

Zeugodacus citrifuscus (Drew \& Romig, 2013). Asia-Pacific. Non-pest.

Zengodacus citroides (Drew, 1989). Asia-Pacific. Non-pest. Cue-lure.

Zeugodacus complicatus (White, 1999). Asia-Pacific. Non-pest. Cue-lure.

Zeugodacus connexus (Hardy, 1982). Asia-Pacific. Non-pest. 
Zengodacus cucumis (French, 1907). Asia-Pacific. Cucurbitaceae fruit pest.

Zengodacus cucurbitae (Coquillett, 1899). Asia-Pacific. Cucurbitaceae fruit pest. Cuelure, zingerone.

Notes: Zeugodacus cucurbitae, the melon fly, is one of the most significant pest species with the Tephritidae. Although different forms are recognized that can be correlated with different hosts, these are generally not thought to represent different (cryptic) species (De Meyer et al. 2015, Hendrichs et al. 2015). Natively widespread in Asia and invasive in many Pacific islands and the Afrotropical region.

Zeugodacus curtus (Drew, 1972). Asia-Pacific. Non-pest. Cue-lure.

Zengodacus daclaciae (Drew \& Romig, 2013). Asia-Pacific. Non-pest. Cue-lure.

Zeugodacus daulus (Drew, 1989). Asia-Pacific. Non-pest. Cue-lure.

Zeugodacus decipiens (Drew, 1972). Asia-Pacific. Cucurbitaceae fruit pest.

Zeugodacus depressus (Shiraki, 1933). Asia-Pacific. Cucurbitaceae fruit pest.

Zeugodacus diaphoropsis (Hering, 1952). Asia-Pacific. Non-pest.

Zeugodacus diaphorus (Hendel, 1915). Asia-Pacific. Non-pest. Cue-lure.

Zeugodacus dissidens (Drew, 1989). Asia-Pacific. Non-pest.

Zeugodacus disturgidus (Yu, Deng \& Chen, 2012). Asia-Pacific. Non-pest.

Notes: $Z$. disturgidus is not included in the Drew and Romig $(2013,2016)$ keys. According to the diagnosis, it is similar to $Z$. vinnulus but differs in having the face with two bands, and the costal band on the wing confluent with vein $\mathrm{R}_{2+3}$ and not expanded apically.

Zeugodacus diversus (Coquillett, 1904). Asia-Pacific. Cucurbitaceae flower pest. Methyl eugenol.

Notes: Drew and Romig (2013) state that this species appears to have a weak attraction to methyl eugenol. We hereby confirm this attraction, based on the recent capture of fifteen flies among eight different trapping locations in Nepal and additional records from Bangladesh.

Zeugodacus dorsirufus (Drew \& Romig, 2013). Asia-Pacific. Non-pest. Cue-lure.

Zeugodacus dubiosus (Hardy, 1982). Asia-Pacific. Non-pest. Cue-lure.

Zeugodacus duplicatus (Bezzi, 1916). Asia-Pacific. Non-pest.

Zeugodacus elegantulus (Hardy, 1974). Asia-Pacific. Non-pest. Cue-lure.

Zeugodacus emarginatus (Perkins, 1939). Asia-Pacific. Non-pest.

Zeugodacus emittens (Walker, 1860). Asia-Pacific. Non-pest. Cue-lure.

Zeugodacus eurylomatus (Hardy, 1982). Asia-Pacific. Non-pest.

Zeugodacus exornatus (Hering, 1941). Asia-Pacific. Non-pest. Cue-lure

Zeugodacus fallacis (Drew, 1972). Asia-Pacific. Non-pest. Cue-lure.

Zeugodacus fereuncinatus (Drew \& Romig, 2013). Asia-Pacific. Non-pest. Cue-lure.

Zeugodacus flavipilosus (Hardy, 1982). Asia-Pacific. Non-pest. Cue-lure.

Zeugodacus flavolateralis (Drew \& Romig, 2013). Asia-Pacific. Non-pest.

Zeugodacus flavopectoralis (Hering, 1953). Asia-Pacific. Non-pest.

Zeugodacus flavoverticalis (Drew \& Romig, 2013). Asia-Pacific. Non-pest. Cue-lure.

Zeugodacus freidbergi (White, 1999). Asia-Pacific. Non-pest. 
Zeugodacus fulvipes (Perkins, 1938). Asia-Pacific. Non-pest.

Zeugodacus fulvoabdominalis (White \& Evenhuis, 1999). Asia-Pacific. Non-pest.

Zeugodacus fuscipennulus (Drew \& Romig, 2001). Asia-Pacific. Non-pest.

Zeugodacus fuscoalatus (Drew \& Romig, 2013). Asia-Pacific. Non-pest.

Zeugodacus gavisus (Munro, 1935). Asia-Pacific. Non-pest. Cue-lure.

Zeugodacus gracilis (Drew, 1972). Asia-Pacific. Non-pest. Cue-lure.

Zeugodacus hamaceki (Drew \& Romig, 2001). Asia-Pacific. Non-pest. Cue-lure.

Zeugodacus hancocki (Drew \& Romig, 2013). Asia-Pacific. Non-pest. Cue-lure.

Zeugodacus hatyaiensis (Drew \& Romig, 2013). Asia-Pacific. Non-pest. Cue-lure.

Zeugodacus havelockiae (Drew \& Romig, 2013). Asia-Pacific. Non-pest. Cue-lure.

Zeugodacus heinrichi (Hering, 1941). Asia-Pacific. Non-pest. Cue-lure, zingerone.

Notes: Zingerone is a new lure record.

Zeugodacus hekouanus (Yu He \& Yang, 2011). Asia-Pacific. Non-pest.

Zeugodacus hengsawadae (Drew \& Romig, 2013). Asia-Pacific. Non-pest.

Zeugodacus hoabinhiae (Drew \& Romig, 2013). Asia-Pacific. Non-pest. Cue-lure

Zeugodacus hochii (Zia, 1936). Asia-Pacific. Cucurbitaceae fruit pest. Cue-lure, zingerone.

Notes: Zingerone is a new lure record.

Zeugodacus hodgsoniae (Drew \& Romig, 2013). Asia-Pacific. Non-pest.

Zeugodacus hoedi (White, 1999). Asia-Pacific. Non-pest.

Zeugodacus hululangatiae (Drew \& Romig, 2013). Asia-Pacific. Non-pest. Cue-lure.

Zengodacus incisus (Walker, 1861). Asia-Pacific. Non-pest. Cue-lure.

Zeugodacus indentus (Hardy, 1974). Asia-Pacific. Non-pest.

Zeugodacus infestus (Enderlein, 1920). Asia-Pacific. Non-pest. Cue-lure.

Zeugodacus iriomotiae (Drew \& Romig, 2013). Asia-Pacific. Non-pest. Methyl eugenol.

Zeugodacus ishigakiensis (Shiraki, 1933). Asia-Pacific. Non-pest. Cue-lure.

Zeugodacus isolatus (Hardy, 1973). Asia-Pacific. Non-pest. Cue-lure.

Zeugodacus javadicus (Mahmood, 1999). Asia-Pacific. Non-pest.

Zeugodacus javanensis (Perkins, 1938), comb. n. Asia-Pacific. Non-pest.

Notes: Originally described in Afrodacus, here transferred from Bactrocera. It is

placed in the subgenus Javadacus. Members of Javadacus were not moved to Zeugodacus by De Meyer et al. (2015) because only one representative, B. unirufa Drew, 1989 , had been included in any molecular phylogenetic studies, where it was robustly placed in Bactrocera. However, B. unirufa has since been synonymized with B. melanothoracica and removed from Javadacus along with several other species that did not have the shallow posterior emargination of sternite $\mathrm{V}$ and elongate posterior surstylus lobes in the male genitalia, which fit Zeugodacus. We therefore now move all remaining species in the subgenus Javadacus to Zeugodacus.

Zeugodacus juxtuncinatus (Drew \& Romig, 2013). Asia-Pacific. Non-pest. Cue-lure. Zeugodacus kaghanae (Mahmood, 1999). Asia-Pacific. Non-pest. Cue-lure.

Zeugodacus khaoyaiae (Drew \& Romig, 2013). Asia-Pacific. Non-pest. Cue-lure. Zeugodacus laguniensis (Drew \& Romig, 2013). Asia-Pacific. Non-pest. Cue-lure. Zeugodacus lipsanus (Hendel, 1915). Asia-Pacific. Non-pest.

Zeugodacus liquidus (Drew \& Romig, 2013). Asia-Pacific. Non-pest. Cue-lure. 
Zeugodacus longicaudatus (Perkins, 1938). Asia-Pacific. Non-pest. Cue-lure.

Zeugodacus longivittatus (Chua \& Ooi, 1998). Asia-Pacific. Non-pest. Methyl eugenol. Zeugodacus luteicinctutus (Ito, 2011). Asia-Pacific. Non-pest.

Notes: Z. luteicinctutus is not included in the Drew and Romig $(2013,2016)$ keys.

According to the diagnosis it is similar to $Z$. yoshimotoi, but differs in having dull

brownish instead of shining a black marking surrounding the ocellar triangle. This

may prove to be a junior synonym of $Z$. yoshimoto $i$ when more specimens are stud-

ied or when molecular data become available.

Zeugodacus macrophyllae (Drew \& Romig, 2013). Asia-Pacific. Non-pest.

Zeugodacus macrovittatus (Drew, 1989). Asia-Pacific. Non-pest. Cue-lure.

Zeugodacus maculatus (Perkins, 1938). Asia-Pacific. Non-pest. Cue-lure.

Zeugodacus maculifacies (Hardy, 1973). Asia-Pacific. Non-pest. Cue-lure.

Zeugodacus maculifemur (Hering, 1938). Asia-Pacific. Non-pest.

Zeugodacus magnicauda (White \& Evenhuis, 1999). Asia-Pacific. Non-pest.

Zeugodacus melanofacies (Drew \& Romig, 2013). Asia-Pacific. Non-pest. Cue-lure.

Zeugodacus melanopsis (Hardy, 1982). Asia-Pacific. Non-pest. Cue-lure.

Zeugodacus menglanus (Yu Liu \& Yang, 2011). Asia-Pacific. Non-pest. Cue-lure.

Zeugodacus mesonotaitha (Drew, 1989). Asia-Pacific. Non-pest.

Zeugodacus minimus (Hering, 1952). Asia-Pacific. Non-pest.

Zeugodacus montanus (Hardy, 1983), comb. nov. Asia-Pacific. Non-pest. Cue-lure.

Notes: Originally described in Dacus, here transferred from Bactrocera. See further comments under $Z$. javanensis.

Zeugodacus mukiae (Drew \& Romig, 2013). Asia-Pacific. Non-pest. Cue-lure.

Zeugodacus mundus (Bezzi, 1919). Asia-Pacific. Cucurbitaceae fruit pest.

Zeugodacus nakhonnayokiae (Drew \& Romig, 2013). Asia-Pacific. Non-pest. Cue-lure. Zeugodacus namlingiae (Drew \& Romig, 2013). Asia-Pacific. Non-pest. Cue-lure.

Zeugodacus neoelegantulus (White, 1999). Asia-Pacific. Non-pest.

Zeugodacus neoemittens (Drew \& Romig, 2013). Asia-Pacific. Non-pest. Cue-lure.

Zeugodacus neoflavipilosus (Drew \& Romig, 2013). Asia-Pacific. Non-pest. Cue-lure.

Zeugodacus neolipsanus (Drew \& Romig, 2013). Asia-Pacific. Non-pest. Cue-lure.

Zeugodacus neopallescentis (Drew, 1989). Asia-Pacific. Non-pest. Cue-lure.

Zeugodacus nigrifacies (Shiraki, 1933). Asia-Pacific. Non-pest.

Zeugodacus ochrosterna (Drew \& Romig, 2013). Asia-Pacific. Non-pest. Cue-lure.

Zeugodacus okunii (Shiraki, 1933). Asia-Pacific. Non-pest.

Zeugodacus pahangiae (Drew \& Romig, 2013). Asia-Pacific. Non-pest.

Zeugodacus pantabanganiae (Drew \& Romig, 2013). Asia-Pacific. Non-pest. Cue-lure.

Zeugodacus papuaensis (Malloch 1939), comb. nov. Asia-Pacific. Non-pest.

Notes: This species was moved from Dacus to the subgenus Austrodacus by Hancock and Drew (2016), but they continued to classify that subgenus in Bactrocera. Like all members of the subgenus Austrodacus, we here place it in the genus Zeugodacus. Zeugodacus paululus (Drew, 1989). Asia-Pacific. Non-pest. Cue-lure.

Zeugodacus pemalangiae (Drew \& Romig, 2013). Asia-Pacific. Non-pest. Cue-lure.

Zeugodacus perplexus (Walker, 1862). Asia-Pacific. Non-pest. 
Zeugodacus perpusillus (Drew, 1971). Asia-Pacific. Non-pest. Cue-lure. Zeugodacus persignatus (Hering, 1941). Asia-Pacific. Non-pest. Cue-lure. Zeugodacus platamus (Hardy, 1973). Asia-Pacific. Non-pest. Cue-lure. Zeugodacus proprescutellatus (Zhang Che \& Gao, 2011). Asia-Pacific. Non-pest. Cue-lure. Zeugodacus pubescens (Bezzi, 1919). Asia-Pacific. Non-pest.

Zeugodacus purus (White, 1999). Asia-Pacific. Non-pest.

Zeugodacus quasiinfestus (Drew \& Romig, 2013). Asia-Pacific. Non-pest. Cue-lure. Zengodacus reflexus (Drew, 1971). Asia-Pacific. Non-pest. Cue-lure.

Zeugodacus rubellus (Hardy, 1973). Asia-Pacific. Non-pest.

Zeugodacus sabahensis (Drew \& Romig, 2013). Asia-Pacific. Non-pest. Cue-lure. Zeugodacus sandaracinus (Drew, 1989). Asia-Pacific. Non-pest.

Zeugodacus sasaotiae (Drew \& Romig, 2013). Asia-Pacific. Non-pest. Cue-lure. Zeugodacus scutellaris (Bezzi, 1913). Asia-Pacific. Cucurbitaceae flower pest. Cue-lure. Zeugodacus scutellarius (Bezzi, 1916), comb. nov. Asia-Pacific. Non-pest. Cue-lure.

Notes: Originally described in Chaetodacus, here transferred from Bactrocera. See further comments under $Z$. javanensis.

Zeugodacus scutellatus (Hendel, 1912). Asia-Pacific. Cucurbitaceae flower pest. Cue-lure. Zeugodacus scutellinus (Bezzi, 1916). Asia-Pacific. Non-pest.

Zeugodacus semisurstyli (Drew \& Romig, 2013), comb. nov. Asia-Pacific. Non-pest. Cue-lure.

Notes: Here transferred from Bactrocera. See further comments under Z. javanensis. Zeugodacus semongokensis (Drew \& Romig, 2013). Asia-Pacific. Non-pest. Cue-lure. Zeugodacus sepikae (Drew, 1989). Asia-Pacific. Non-pest. Zeugodacus signatifer (Tryon, 1927). Asia-Pacific. Non-pest. Zeugodacus signatus (Hering, 1941). Asia-Pacific. Non-pest. Cue-lure. Zeugodacus sinensis (Yu Bai \& Chen, 2011). Asia-Pacific. Non-pest. Cue-lure. Zeugodacus singularis (Drew, 1989). Asia-Pacific. Non-pest. Cue-lure. Zeugodacus sonlaiae (Drew \& Romig, 2013). Asia-Pacific. Non-pest. Cue-lure. Zeugodacus speciosus (Drew \& Romig, 2013). Asia-Pacific. Non-pest. Cue-lure. Zeugodacus spectabilis (Drew \& Romig, 2013). Asia-Pacific. Non-pest. Cue-lure. Zengodacus strigifinis (Walker, 1861). Asia-Pacific. Cucurbitaceae flower pest. Cue-lure. Zeugodacus sumbensis (Hering, 1953). Asia-Pacific. Non-pest.

Zeugodacus surrufulus (Drew, 1989). Asia-Pacific. Non-pest. Cue-lure. Zeugodacus synnephes (Hendel, 1913). Asia-Pacific. Non-pest. Cue-lure. Zeugodacus tapervitta (Mahmood, 1999). Asia-Pacific. Cucurbitaceae fruit pest. Zeugodacus tappanus (Shiraki, 1933). Asia-Pacific. Non-pest.

Zengodacus tan (Walker, 1849). Asia-Pacific. Cucurbitaceae fruit pest. Cue-lure.

Notes: Zeugodacus tau possibly represents a cryptic species complex the extent of which is currently unclear (Baimai 2000, Kitthawee and Dujardin 2010, Kitthawee and Rungsri 2011, Dujardin and Kitthawee 2013).

Zeugodacus tebeduiae (Drew \& Romig, 2013). Asia-Pacific. Non-pest. Cue-lure. Zeugodacus timorensis (Perkins, 1939). Asia-Pacific. Non-pest. Cue-lure. 
Zeugodacus transversus (Hardy, 1982). Asia-Pacific. Non-pest. Cue-lure.

Zeugodacus triangularis (Drew, 1968). Asia-Pacific. Cucurbitaceae flower pest. Cuelure, zingerone.

Zeugodacus trichosanthes (Drew \& Romig, 2013). Asia-Pacific. Cucurbitaceae fruit pest. Cue-lure.

Zeugodacus trichotus (May, 1962). Asia-Pacific. Non-pest. Cue-lure.

Zeugodacus tricuspidatae (Drew \& Romig, 2013). Asia-Pacific. Non-pest.

Zeugodacus trilineatus (Hardy, 1955), comb. nov. Asia-Pacific. Non-pest. Cue-lure.

Notes: Originally described in Dacus, here transferred from Bactrocera. See further comments under $Z$. javanensis.

Zeugodacus trimaculatus (Hardy \& Adachi, 1954). Asia-Pacific. Cucurbitaceae fruit pest. Zeugodacus trivandrumensis (Drew \& Romig, 2013). Asia-Pacific. Non-pest.

Zeugodacus ujungpandangiae (Drew \& Romig, 2013). Asia-Pacific. Non-pest. Cue-lure. Zeugodacus uncinatus (Drew \& Romig, 2013). Asia-Pacific. Non-pest. Cue-lure.

Zeugodacus unilateralis (Drew, 1989). Asia-Pacific. Non-pest.

Zeugodacus univittatus (Drew, 1972). Asia-Pacific. Non-pest. Cue-lure.

Zeugodacus urens (White, 1999). Asia-Pacific. Non-pest.

Zeugodacus vargus (Hardy, 1982). Asia-Pacific. Non-pest. Cue-lure.

Zeugodacus vinnulus (Hardy, 1973). Asia-Pacific. Non-pest. Cue-lure.

Zeugodacus vultus (Hardy, 1973). Asia-Pacific. Non-pest. Cue-lure

Zengodacus waimitaliae (Drew \& Romig, 2013). Asia-Pacific. Non-pest. Cue-lure.

Zeugodacus watersi (Hardy, 1954). Asia-Pacific. Non-pest.

Zeugodacus whitei (Drew \& Romig, 2013). Asia-Pacific. Non-pest. Cue-lure.

Zeugodacus yalaensis (Drew \& Romig, 2013). Asia-Pacific. Non-pest.

Zeugodacus yoshimotoi (Hardy, 1973). Asia-Pacific. Non-pest. Cue-lure.

Zeugodacus zahadi (Mahmood, 1999). Asia-Pacific. Non-pest. Cue-lure.

Notes: The characters that supposedly distinguish $Z$. zahadi from $Z$. tau overlap, and $Z$. zahadi may be a synonym of $Z$. tau (Drew \& Romig, 2013). See further notes under $Z$. tau.

\section{Acknowledgements}

We would like to thank Ho-Yeon Han, one anonymous reviewer, and the editor for their comments, which helped to improve the manuscript. This research was funded in part by the College of Tropical Agriculture and Human Resources, University of Hawaii, at Manoa, the USDA Cooperative State Research, Education and Extension (CSREES) project HAW00942-H administered by the College of Tropical Agriculture and Human Resources, University of Hawaii, and the USDA Farm Bill grant, FY17 3.0497.01. Mention of trade names or commercial products in this publication is solely for the purpose of providing specific information and does not imply recommendation or endorsement. USDA is an equal opportunity provider and employer. 


\section{References}

Baimai V (2000) Cytological evidence for a complex of species within the taxon Bactrocera tau (Diptera: Tephritidae) in Thailand. Biological Journal of the Linnean Society 69: 399-409. https://doi.org/10.1006/bijl.1999.0377

CABI (2017) Invasive Species Compendium. http://www.cabi.org/isc/ [accessed Oct. 2017]

Cameron EC, Sved JA, Gilchrist AS (2010) Pest fruit fly (Diptera: Tephritidae) in northwestern Australia: One species or two? Bulletin of Entomological Research 100: 197-206. https://doi.org/10.1017/S0007485309990150

Clarke AR, Armstrong KF, Carmichael AE, Milne JR, Raghu S, Roderick GK, Yeates DK (2005) Invasive phytophagous pests arising through a recent tropical evolutionary radiation: the Bactrocera dorsalis complex of fruit flies. Annual Review of Entomology 50: 293-319. https://doi.org/10.1146/annurev.ento.50.071803.130428

David KJ, Hancock DL, Singh SK, Ramani S, Behere GT, Salini S (2017) New species, new records and updated subgeneric key of Bactrocera Macquart (Diptera: Tephritidae: Dacinae: Dacini) from India. Zootaxa 4272: 386-400. https://doi.org/10.11646/zootaxa.4272.3.4

David KJ, Ramani S, Whitmore D, Ranganath HR (2016) Two new species and a new record of Bactrocera Macquart (Diptera: Tephritidae: Dacinae: Dacini) from India. Zootaxa 4103: 25-34. https://doi.org/10.11646/zootaxa.4103.1.2

De Meyer M, Delatte H, Mwatawala M, Quilici S, Vayssières JF, Virgilio M (2015) A review of the current knowledge on Zeugodacus cucurbitae (Coquillett) (Diptera, Tephritidae) in Africa, with a list of species included in Zeugodacus. ZooKeys 540: 539-557 + Supplementary material 1:(4). https://doi.org/10.3897/zookeys.540.9672

De Meyer M, White I (2016) True Fruit Flies (Diptera, Tephritidae) of the Afrotropical Region. http://projects.bebif.be/fruitfly/index.html [accessed Oct. 2017]

Drew RAI (1971) New species of Dacinae (Diptera: Trypetidae) from the South Pacific Area. Queensland Journal of Agricultural Science 28: 29-103.

Drew RAI (1989) The tropical fruit flies (Diptera: Tephritidae: Dacinae) of the Australasian and Oceanian Regions. Memoirs of the Queensland Museum 1: 1-536.

Drew RAI, Hancock DL (1994) The Bactrocera dorsalis complex of fruit flies (Diptera: Tephritidae: Dacinae) in Asia. Bulletin of Entomological Research Supplement Series 2: 1-68. https://doi.org/10.1017/S1367426900000278

Drew RAI, Hancock DL (2016) A review of the subgenus Bulladacus Drew \& Hancock of Bactrocera Macquart (Diptera: Tephritidae: Dacinae), with description of two new species from Papua New Guinea. Australian Entomologist 43: 189-210.

Drew RAI, Ma J, Smith S, Hughes JM (2011) The taxonomy and phylogenetic relationships of species in the Bactrocera musae complex of fruit flies (Diptera: Tephritidae: Dacinae) in Papua New Guinea. Raffles Bulletin of Zoology 59: 145-162.

Drew RAI, Romig MC (2013) Tropical fruit flies of South-East Asia. CABI, Wallingford, 655 pp.

Drew RAI, Romig MC (2016) Keys to the Tropical Fruit Flies of South-East Asia. CABI, Wallingford, $487 \mathrm{pp}$.

Dujardin JP, Kitthawee S (2013) Phenetic structure of two Bactrocera tau cryptic species (Diptera: Tephritidae) infesting Momordica cochinchinensis (Cucurbitaceae) in Thailand and Laos. Zoology 116: 129-138. https://doi.org/10.1016/j.zool.2012.07.004 
Dupuis JR, Bremer FT, Kauwe A, San Jose M, Leblanc L, Rubinoff D, Geib S (2017) HiMAP: Robust phylogenomics from highly multiplexed amplicon sequencing. bioRxiv. http://biorxiv.org/content/early/2017/11/05/213454.abstract

Ebina T, Ohto K (2006) Morphological characters and PCR-RFLP markers in the interspecific hybrids between Bactrocera carambolae and B. papayae of the B. dorsalis species complex (Diptera: Tephritidae). Research Bulletin of the Plant Protection Service, Japan 42: 23-34.

Ekesi S, De Meyer M, Mohamed SA, Virgilio M, Borgemeister C (2016) Taxonomy, ecology, and management of native and exotic fruit fly species in Africa. Annual Review of Entomology 61: 219-238. https://doi.org/10.1146/annurev-ento-010715-023603

Fay HAC (2012) A highly effective and selective male lure for Bactrocera jarvisi (Tryon) (Diptera: Tephritidae). Australian Journal of Entomology 51: 189-197. https:/doi. org/10.1111/j.1440-6055.2011.00847.x

Fletcher B (1987) The biology of Dacine fruit flies. Annual Review of Entomology 32: 115-144. https://doi.org/10.1146/annurev.ento.32.1.115

Freidberg A, Kovac D, Shiao S (2017) A revision of Ichneumonopsis Hardy, 1973 (Diptera: Tephritidae: Dacinae: Gastrozonini ), Oriental bamboo-shoot fruit flies. European Journal of Taxonomy 317: 1-23. https://doi.org/10.5852/ejt.2017.317

Gilchrist AS, Wang Y, Yu H, Raphael K (2003) Genetic delineation of sibling species of the pest fruit fly Bactocera (Diptera: Tephritidae) using microsatellites. Bulletin of Entomological Research 93: 351-360. https://doi.org/10.1079/BER2003249

Han H-Y, Choi D-S, Ro K-E (2017) Taxonomy of Korean Bactrocera (Diptera: Tephritidae: Dacinae) with review of their biology. Journal of Asia-Pacific Entomology 20: 1321-1332. https://doi.org/10.1016/j.aspen.2017.09.011

Hancock DL (2015) A new subgenus for six Indo-Australian species of Bactrocera Macquart (Diptera: Tephritidae: Dacinae) and subgeneric transfer of four other species. Australian Entomologist 42: 39-44.

Hancock DL, Hamacek EL, Lloyd AC, Elson-Harris MM (2000) The distribution and host plants of fruit flies (Diptera: Tephritidae) in Australia. Information Series QI99067, Queensland Department of Primary Industries, Brisbane, 75 pp.

Hancock DL, Drew RAI (2006) A revised classification of subgenera and species groups in Dacus Fabricius (Diptera: Tephritidae). Instrumenta Biodiversitatis 7: 167-205.

Hancock DL, Drew RAI (2015) A review of the Indo-Australasian subgenus Parazeugodacus Shiraki of Bactrocera Macquart (Diptera: Tephritidae: Daciniae). Australian Entomologist 42: 91-104.

Hancock DL, Drew RAI (2017a) A review of the subgenus Javadacus Hardy of Bactrocera Macquart (Diptera: Tephritidae: Dacinae). Australian Entomologist 44: 105-112.

Hancock DL, Drew RAI (2017b) A review of the Indo-Australian subgenera Heminotodacus Drew, Paradacus Perkins and Perkinsidacus subgen. n. of Bactrocera Macquart (Diptera: Tephritidae: Dacinae). Australian Entomologist 44: 137-146.

Hardy DE (1954) The Dacus subgenera Neodacus and Gymnodacus of the world (Diptera, Tephritidae). Proceedings of the Entomological Society of Washington 56: 5-23.

Hardy DE (1955) A reclassification of the Dacini (Tephritidae-Diptera). Annals of the Entomological Society of America 48: 1-13. https://doi.org/10.1093/aesa/48.6.425 
Hardy DE (1976) Resurrection of Bactrocera Macquart and clarification of the type-species, longicornis Macquart (Diptera: Tephritidae). Proceedings of the Hawaiian Entomological Society 22: 245-249.

Hendel FG (1927) Einige neue Bohrfliegen (Trypetidae) aus dem Hamburger Museum. Wiener Entomologische Zeitung 44: 58-65.

Hendrichs J, Vera MT, De Meyer M, Clarke AR (2015) Resolving cryptic species complexes of major tephritid pests. ZooKeys 540: 5-39. https://doi.org/10.3897/zookeys.540.9656

International Commission on Zoological Nomenclature (1999) International Code of Zoological Nomenclature. Fourth edition. In: Ride WDL et al. (Eds) The International Trust for Zoological Nomenclature, London, 306 pp. http://www.nhm.ac.uk/hosted-sites/iczn/code/ Ito S (2011) Die Bohrfliegen aus Nordost-Nepal (Diptera, Tephritidae). Esakia 51: 1-45.

Kitthawee S, Dujardin JP (2010) The geometric approach to explore the Bactrocera tau complex (Diptera: Tephritidae) in Thailand. Zoology 113: 243-249. https://doi.org/10.1016/j. zool.2009.12.002

Kitthawee S, Rungsri N (2011) Differentiation in wing shape in the Bactrocera tau (Walker) complex on a single fruit species of Thailand. ScienceAsia 37: 308-313. https://doi. org/10.2306/scienceasia1513-1874.2011.37.308

Krosch MN, Schutze MK, Armstrong KF, Graham GC, Yeates DK, Clarke AR (2012) A molecular phylogeny for the Tribe Dacini (Diptera: Tephritidae): systematic and biogeographic implications. Molecular Phylogenetics and Evolution 64: 513-523. https://doi.org/10.1016/j.ympev.2012.05.006

Leblanc L, San Jose M, Rubinoff D (2015a) Description of a new species and new country distibution records of Bactrocera (Diptera: Tephritidae: Dacinae) from Cambodia. Zootaxa 4012: 593-600. https://doi.org/10.11646/zootaxa.4012.3.12

Leblanc L, San Jose M, Barr N, Rubinoff D (2015b) A phylogenetic assessment of the polyphyletic nature and intraspecific color polymorphism in the Bactrocera dorsalis complex (Diptera, Tephritidae). ZooKeys 540: 339-367. https://doi.org/10.3897/zookeys.540.9786

Manrakhan A, Daneel JH, Beck R, Virgilio M, Meganck K, De Meyer M (2017) Efficacy of trapping systems for monitoring of Afrotropical fruit flies. Journal of Applied Entomology. https://doi.org/10.1111/jen.12373

Munro HK (1984) A taxonomic treatise on the Dacidae (Tephritoidea, Diptera) of Africa. Entomology Memoirs, Department of Agriculture and Water Supply, Republic of South Africa 61: 1-313.

Nakahara SMM, Muraji M (2008) Phylogenetic analyses of Bactrocera fruit flies (Diptera: Tephritidae) based on nucleotide sequences of the mitochondrial COI and COII genes. Research Bulletin of the Plant Protection Service Japan 44: 1-12.

Norrbom AL, Carroll LE, Thompson FC, White IM, Freidberg A (1999) Systematic Database of Names. In: Thompson FC (Ed.) Fruit fly expert identification system and systematic information database. Myia (1998) 9: 65-251, \& Diptera Data Dissemination Disk (CD-ROM) (1998) 1.

Norrbom AL (2004) Updates to Biosystematic Database of World Diptera for Tephritidae through 1999. Diptera Data Dissemination Disk (CD-ROM) 2.

Pape T, Blagoderov V, Mostovski MB (2011) Order DIPTERA Linnaeus, 1758. Zootaxa 3148: 222-229. 
Pape T, Thompson FC (Eds) (2013) Systema Diptorum, Version 1.5. http://diptera.org/ [accessed on Oct. 2018]

Pest Management in the Pacific Project (2003) Pacific Fruit Fly Project. https://lrd.spc.int/ pacific-fruit-fly [accessed on Oct. 2017]

Roskov Y, Abucay L, Orrell T, Nicolson D, Bailly N, Kirk PM, Bourgoin T, DeWalt RE, Decock W, De Wever A, Nieukerken EJ van, Zarucchi J, Penev L (2017) Species 2000 \& ITIS Catalogue of Life, 2017 Annual Checklist. Digital resource at www.catalogueoflife.org/ annual-checklist/2017. Species 2000: Naturalis, Leiden, the Netherlands. http://projects. bebif.be/fruitfly/index.html

Royer JE (2015) Responses of fruit flies (Tephritidae: Dacinae) to novel male attractants in north Queensland, Australia, and improved lures for some pest species. Austral Entomology 54: 411-426. https://doi.org/10.1111/aen.12141

Royer JE, Agovaua S, Bokosou J, Kurika K, Mararuai A, Mayer DG, Niangu B (2017) Responses of fruit flies (Diptera: Tephritidae) to new attractants in Papua New Guinea. Austral Entomology. https://doi.org/10.1111/aen.12269

San Jose M, Doorenweerd C, Leblanc L, Barr N, Geib SM, Rubinoff D (2018) Incongruence between molecules and morphology: A seven-gene phylogeny of Dacini fruit flies paves the way for reclassification (Diptera: Tephritidae). Molecular Phylogenetics and Evolution. https://doi.org/10.1016/j.ympev.2017.12.001

San Jose M, Leblanc L, Rubinoff D (2013) An evaluation of the species status of Bactrocera invadens and the systematics of the Bactrocera dorsalis (Diptera: Tephritidae) complex. Annals of the Entomological Society of America 106: 684-694. https://doi.org/10.1603/AN13017

Schutze MK, Aketarawong N, Amornsak W, Armstrong KF, Augustinos AA, Barr N, Bo W, Bourtzis K, Boykin LM, Cáceres C, Cameron SL, Chapman TA, Chinvinijkul S, Chomic A, De Meyer M, Drosopoulou E, Englezou A, Ekesi S, Gariou-Papalexiou A, Geib SM, Hailstones D, Hasanuzzaman M, Haymer D, Hee AKW, Hendrichs J, Jessup A, Ji Q, Khamis FM, Krosch MN, Leblanc LUC, Mahmood K, Malacrida AR, Mavragani-Tsipidou P, Mwatawala M, Nishida R, Ono H, Reyes J, Rubinoff D, San Jose M, Shelly TE, Srikachar S, Tan KH, Thanaphum S, Haq I, Vijaysegaran S, Wee SL, Yesmin F, Zacharopoulou A, Clarke AR (2015a) Synonymization of key pest species within the Bactrocera dorsalis species complex (Diptera: Tephritidae): Taxonomic changes based on a review of 20 years of integrative morphological, molecular, cytogenetic, behavioural and chemoecological data. Systematic Entomology 40: 456-471. https://doi.org/10.1111/syen.12113

Schutze MK, Mahmood K, Pavasovic A, Bo W, Newman J, Clarke AR, Krosch MN, Cameron SL (2015b) One and the same: Integrative taxonomic evidence that Bactrocera invadens (Diptera: Tephritidae) is the same species as the Oriental fruit fly Bactrocera dorsalis. Systematic Entomology 40: 472-486. https://doi.org/10.1111/syen.12114

Schutze MK, Virgilio M, Norrbom A, Clarke AR (2017) Tephritid integrative taxonomy: Where we are now, with a focus on the resolution of three tropical fruit fly species complexes. Annual Review of Entomology 62: 147-164. https://doi.org/10.1146/annurev-ento-031616-035518

Smith PT, Kambhampati S, Armstrong KA (2003) Phylogenetic relationships among Bactrocera species (Diptera: Tephritidae) inferred from mitochondrial DNA sequences. Molecular Phylogenetics and Evolution 26: 8-17. https://doi.org/10.1016/S1055-7903(02)00293-2 
Tan KH, Nishida R (2000) Mutual reproductive benefits between a wild orchid, Bulbophyllum patens, and Bactrocera fruit flies via a floral synomone. Journal of Chemical Ecology 26: 533-546. https://doi.org/10.1023/A:1005477926244

Vargas RI, Pinero JC, Leblanc L (2015) An overview of pest species of Bactrocera fruit flies (Diptera: Tephritidae) and the integration of biopesticides with other biological approaches for their management with a focus on the Pacific region. Insects 6: 297-318. https://doi.org/10.3390/insects6020297

Virgilio M, Jordaens K, Verwimp C, White IM, De Meyer M (2015) Higher phylogeny of frugivorous flies (Diptera, Tephritidae, Dacini): Localised partition conflicts and a novel generic classification. Molecular Phylogenetics and Evolution 85: 171-179. https://doi. org/10.1016/j.ympev.2015.01.007

White IM (1999) Morphological features of the Tribe Dacini (Dacinae): Their significance to behavior and classification. In: Aluja M, Norrbom AL (Eds) Fruit Flies (Tephritidae): Phylogeny and evolution of Behavior. CRC Press, Boca Raton, 505-534. https://doi. org/10.1201/9781420074468.ch20

White IM (2006) Taxonomy of the Dacina (Diptera:Tephritidae) of Africa and the Middle East. African Entomology Memoir 2: 1-156.

White IM, Headrick DH, Norrbom AL, Carroll LE (1999) Glossary. In: Aluja M, Norrbom AL (Eds) Fruit flies (Tephritidae): Phylogeny and evolution of behavior. CRC Press, Boca Raton, 881-924. https://doi.org/10.1201/9781420074468.sec8

White IM, Elson-Harris MM (1992) Fruit flies of economic significance. CABI, Wallingford, 601 pp. Whitman DW, Orsak L, Greene E (1988) Spider mimicry in fruit flies (Diptera: Tephritidae): Further experiments on the deterrence of jumping spiders (Araneae: Salticidae) by Zonosemata vittigera (Coquillett). Annals of the Entomological Society of America 81: 532-536. http://dx.doi.org/10.1093/aesa/81.3.532

Yu H, Deng Y-L, Chen N-Z (2012) A new species of the subgenus Sinodacus from Yunnan, China (Diptera, Tephritidae). Acta Zootaxonomica Sinica 37: 834-836.

Zhang Z-Q (2011) Animal biodiversity: An outline of higher-level classification and survey of taxonomic richness. Zootaxa 3148: 1-237.

\section{Supplementary material I}

\section{A global checklist of the 932 fruit fly species in the tribe Dacini (Diptera: Teph- ritidae), spreadsheet table.}

Authors: Camiel Doorenweerd, Luc Leblanc, Allen L. Norrbom, Michael San Jose, Daniel Rubinoff

Data type: Checklist

Copyright notice: This dataset is made available under the Open Database License (http://opendatacommons.org/licenses/odbl/1.0/). The Open Database License $(\mathrm{ODbL})$ is a license agreement intended to allow users to freely share, modify, and use this Dataset while maintaining this same freedom for others, provided that the original source and author(s) are credited.

Link: https://doi.org/10.3897/zookeys.730.21786.suppl1 